\title{
NARROWBAND IMAGING OF ESCAPING LYMAN-CONTINUUM EMISSION IN THE SSA22 FIELD*,†
}

\author{
Daniel B. Nestor ${ }^{1}$, Alice E. Shapley ${ }^{1,3}$, Charles C. Steidel ${ }^{2}$, And Brian Siana ${ }^{2}$ \\ ${ }^{1}$ Department of Astronomy, University of California, Los Angeles, 430 Portola Plaza, Los Angeles, CA 90024, USA \\ ${ }^{2}$ California Institute of Technology, MS 105-24, Pasadena, CA 91125, USA \\ Received 2011 January 31; accepted 2011 April 26; published 2011 June 29
}

\begin{abstract}
We present the results of an ultradeep, narrowband imaging survey for Lyman-continuum (LyC) emission at $z \sim 3$ in the SSA22a field. We employ a custom narrowband filter centered at $\lambda=3640 \AA$ (NB3640), which probes the LyC region for galaxies at $z \geqslant 3.06$. We also analyze new and archival NB4980 imaging tuned to the wavelength of the Ly $\alpha$ emission line at $z=3.09$, and archival broadband $B, V$, and $R$ images of the non-ionizing UV continuum. Our NB3640 images contain $26 z \geqslant 3.06$ Lyman break galaxies (LBGs) as well as a set of 130 Ly $\alpha$ emitters (LAEs), identified by their excess NB4980 flux relative to the $B V$ continuum. Six LBGs and 28 LAEs are detected in the NB3640 image. LBGs appear to span a range of NB3640- $R$ colors, while LAEs appear bimodal in their NB3640 $R$ properties. We estimate average UV-to-LyC flux density ratios, corrected for foreground contamination and intergalactic medium absorption, finding $\left\langle F_{\mathrm{UV}} / F_{\mathrm{LyC}}\right\rangle_{\text {corr }}^{\mathrm{LBG}}=11.3_{-5.4}^{+10.3}$, which implies an LBG LyC escape fraction $f_{\text {esc }}^{\mathrm{LyC}} \sim 0.1$, and $\left\langle F_{\mathrm{UV}} / F_{\mathrm{LyC}}\right\rangle_{\text {corr }}^{\mathrm{LAE}}=2.2_{-0.6}^{+0.9}$. The strikingly blue LAE flux density ratios defy interpretation in terms of standard stellar population models. Assuming $\left\langle F_{\mathrm{UV}} / F_{\mathrm{LyC}}\right\rangle_{\text {corr }}^{\mathrm{LBG}}$ applies down to $L=0.1 L^{*}$, we estimate a galaxy contribution to the intergalactic hydrogen ionization rate that is consistent with independent estimates based on the Ly $\alpha$ forest opacity at $z \simeq 3$. If we assume that $\left\langle F_{\mathrm{UV}} / F_{\mathrm{LyC}}\right\rangle_{\text {corr }}^{\mathrm{LAE}}$ holds at the faintest luminosities, the galaxy contribution significantly exceeds that inferred from the Ly $\alpha$ forest. We interpret our results in terms of a model where LyC photons escape over only $\sim 10 \%-20 \%$ of solid angle. When advantageously oriented, a galaxy will exhibit a low UV-to-LyC ratio, an effect enhanced for more compact galaxies. This model, however, does not adequately explain the extremely blue NB3640- $R$ colors measured for some LAEs in our sample. Further follow-up study of these faint LAEs is crucial, given the potentially important contribution similar objects make to the process of reionization.
\end{abstract}

Key words: cosmology: observations - diffuse radiation - galaxies: high-redshift - intergalactic medium

Online-only material: color figures

\section{INTRODUCTION}

The thermal state of the intergalactic medium (IGM) is governed by its spatial density distribution, the spectral shape and intensity of the metagalactic ionizing background, and the distribution of the sources of the background. Identifying these sources constitutes a critical step toward understanding the physics and thermal history of the IGM. Quasi-stellar objects (QSOs) are efficient producers of far-UV radiation and dominate the Lyman-continuum ( $\mathrm{LyC}$ ) emissivity that maintains the ionization state of the IGM up to the peak of active galactic nucleus activity at redshift $z \sim 2$ (Cowie et al. 2009). Beyond $z \sim 2$, however, their contribution decreases drastically with their decreasing number density (Hopkins et al. 2007). As it has been demonstrated that reionization was completed by $z \gtrsim 6$ (Fan et al. 2006; Becker et al. 2007), it is generally assumed that the ionizing flux required for reionization was produced in starforming galaxies. Direct measurements of ionizing radiation escaping from galaxies at $z \gtrsim 6$ are not possible because of the large optical depth to LyC photons in the high-redshift IGM. However, the measurement can in principle be made at lower redshift where the IGM is less opaque.

\footnotetext{
* Based, in part, on data obtained at the W. M. Keck Observatory, which is operated as a scientific partnership among the California Institute of Technology, the University of California, and NASA, and was made possible by the generous financial support of the W. M. Keck Foundation.

$\dagger$ Based, in part, on data collected at the Subaru Telescope and obtained from the SMOKA archive, which is operated by the Astronomy Data Center, National Astronomical Observatory of Japan.

3 David and Lucile Packard Fellow.
}

Although observations of star-forming galaxies at relatively low redshift $(0.5 \lesssim z \lesssim 2)$ have failed to detect escaping LyC photons (e.g., Malkan et al. 2003; Siana et al. 2007; Grimes et al. 2009; Siana et al. 2010; Bridge et al. 2010), there has been much recent progress in the search for $\mathrm{LyC}$ emission at higher $(z \sim 3-4)$ redshift. Building on the initial detection by Steidel et al. (2001) in a composite spectrum of 29 LBGs at $\langle z\rangle=3.4$, Shapley et al. (2006) presented the first spectroscopic detections of LyC emission from individual objects. In a sample of 14 objects with deep Keck/LRIS spectra, two exhibited significant LyC emission, implying $f_{\text {esc }}^{\mathrm{LyC}}>0.15$. Deep Keck/ LRIS spectroscopy for a $z \sim 3$ sample larger by an order of magnitude is forthcoming, and can be used to generalize the initial spectroscopic results (C. C. Steidel et al. 2011, in preparation).

An alternative technique for studying the escape of LyC emission consists of narrowband imaging. Measuring the density of LyC radiation with imaging requires a filter that only transmits flux below the Lyman limit, and also one that is relatively narrow. A filter covering too broad a range below the Lyman limit will indicate more about the statistics of IGM absorption than the absorption intrinsic to galaxies. Specifically, a filter width comparable to or larger than the wavelength range corresponding to one LyC photon mean free path will typically contain significant absorption due to intervening Lyman limit systems, thus making it more difficult to infer the amount of escaping ionizing radiation in the immediate vicinity of a highredshift galaxy. Current estimates of the mean free path at $z \sim 3$ are $\sim 70-85 \mathrm{Mpc}$ (proper), assuming the current concordance 
cosmology (Faucher-Giguère et al. 2008; Prochaska et al. 2009; Songaila \& Cowie 2010). This range in mean free path corresponds to a rest-frame wavelength interval of $\sim 850-910 \AA$. Since standard broadband $U$ filters probe a larger wavelength baseline than this, it is advantageous to adopt a custom setup, including a narrowband filter whose transmission curve probes within the rest wavelength range corresponding to one mean free path.

Key for maximizing the efficiency of narrowband imaging in a fixed rest-frame wavelength range is the existence of a large sample of galaxies in a single field at roughly the same redshift. In this respect, the SSA22a field serves as an ideal target for narrowband imaging searches for LyC emission at $z \sim 3$. This field has been shown to contain a significant overdensity of Lyman break galaxies (LBGs) at $z=3.09 \pm 0.03$ (Steidel et al. 1998). In addition to the 27 LBGs spectroscopically confirmed to lie within the $z=3.09$ redshift spike, narrowband imaging tuned to the wavelength of $\operatorname{Ly} \alpha$ emission at $z=3.09$ has been used to identify large samples of Ly $\alpha$ emitters (LAEs) and extended Ly $\alpha$ "blobs" at the same redshift (Steidel et al. 2000; Matsuda et al. 2004; Hayashino et al. 2004).

Escaping LyC emission at $z \geqslant 3$ detected by narrowband imaging was first reported by Iwata et al. (2009). Using Subaru/ Suprime-Cam and a "NB359" filter, with a central wavelength $3590 \AA$, and FWHM of $150 \AA$, these authors searched for LyC emission for sources at $z \geqslant 3.06$, and reported detections for 7 LBGs and 10 LAEs. In some cases there are spatial offsets of several kpc between the centroids of ionizing and non-ionizing UV emission, which may distinguish among different models for the escape of LyC emission (e.g., Gnedin et al. 2008). Furthermore, in some of the fainter LAEs, the high apparent ratio of escaping ionizing to non-ionizing UV radiation challenges standard models for the intrinsic spectral energy distribution of star-forming galaxies (Inoue 2010; Inoue et al. 2011). In a parallel and independent experiment, we have used Keck/LRIS and a narrowband "NB3640" filter, similar to the Suprime-Cam NB359 filter, in order to collect even deeper images of the LyC region for $z \sim 3$ galaxies in the SSA22a field. We have also obtained significantly deeper imaging tuned to the wavelength of $\operatorname{Ly} \alpha$, enabling more robust identification of LAEs, down to fainter flux limits.

In this paper, we present the results of our narrowband imaging survey for LyC emission at $z \sim 3$ in the SSA22a field. Section 2 describes the observations and data reduction. In Section 3, we present narrow and broadband photometric measurements, the identification of a sample of LAEs based on our new, deeper data set, and the method used for matching apparent LyC detections with known $z \sim 3$ targets. We also discuss potential emission contamination along the line of sight in our narrowband imaging. Section 4 contains our results, including estimates of the true ratios of ionizing to non-ionizing flux densities for our targets, the corresponding escape fractions, and the implied space density of ionizing radiation. In Section 5, we discuss the cosmological implications of these results, and offer a concluding summary in Section 6. Throughout the paper we employ the $\mathrm{AB}$ magnitude system, quote flux and luminosity densities in units of $F_{v}$ and $L_{v}$, respectively, and assume a cosmology with $\Omega_{m}=0.3, \Omega_{\Lambda}=0.7$, and $H_{0}=70 \mathrm{~km} \mathrm{~s}^{-1} \mathrm{Mpc}^{-1}$.

\section{OBSERVATIONS AND DATA REDUCTION}

A custom narrowband filter was used for LyC observations, with central wavelength $3635 \AA$ and FWHM $100 \AA$, hereafter

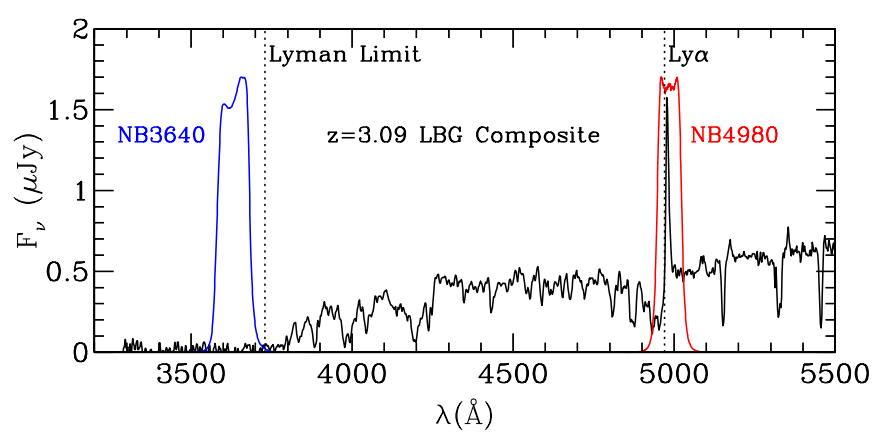

Figure 1. Narrowband filter transmission curves overlaid on an LBG composite spectrum from Shapley et al. (2006), with $\langle z\rangle=3.09$. The NB3640 filter probes the LyC region for galaxies at $z \geqslant 3.06$, while the NB4980 filter contains the Ly $\alpha$ feature for galaxies at $z \sim 3.09$, the redshift of the significant galaxy overdensity in the SSA22a field. Dotted lines mark both the Lyman limit and Ly $\alpha$ feature at $z=3.09$.

(A color version of this figure is available in the online journal.)

referred to as NB3640. The filter was originally designed to detect Ly $\alpha$ emission at $z=2$ (Andrew Blain 2005, private communication), but its specifications are such that no significant contamination $(\leqslant 1 \%)$ longward of the Lyman limit $(912 \AA)$ will enter the filter for redshifts $z \geqslant 3.06$. Furthermore, the narrow NB3640 bandpass probes the wavelength region well within one mean free path below the Lyman limit at the redshift for the majority of our target galaxies (Faucher-Giguère et al. 2008; Prochaska et al. 2009; Songaila \& Cowie 2010), thus minimizing the correction for IGM absorption that is required to infer $f_{\mathrm{esc}}^{\mathrm{LyC}}$ (Shapley et al. 2006). Figure 1 shows the transmission curve of the NB3640 filter overlaid on a composite $z=3.09 \mathrm{LBG}$ spectrum from Shapley et al. (2006). Also shown in Figure 1 is the transmission curve of an additional custom narrowband filter tuned to the wavelength of $\operatorname{Ly} \alpha$ at $z=3.09$, the SSA22a spike redshift. This filter has central wavelength $4985 \AA$ and FWHM $80 \AA$, hereafter referred to as NB4980.

We obtained NB3640 imaging data using the blue side of the Low Resolution Imaging Spectrometer (LRIS; Steidel et al. 2004). The NB3640 imaging data were collected on 2005 August 6-7 and 2008 June 2-4 (UT), for a total of $13.2 \mathrm{hr}$ of integration. During the 2005 August run, the red side of LRIS was simultaneously used to collect NB4980 data, for a total integration time of $9.4 \mathrm{hr}$. The blue side (LRIS-B) detector has a plate scale of 0.135 , while the red side (LRIS-R) has 0.211 pixels. The "d460" dichroic beam splitter was used to send light bluer than $\sim 4600 \AA$ toward the LRIS-B, while longer wavelengths were directed toward LRIS-R. Conditions were photometric during both observing runs, with seeing in the final NB3640 and NB4980 stacked images of 0.80 . The pointing center of the LRIS imaging field was $\alpha=22: 17: 28.8$, $\delta=+00: 14: 36$, and the area of the final trimmed stacks is $5.5 \times 7^{\prime} \cdot 6$. Contained within this footprint are 26 of the 33 LBGs in SSA22a with spectroscopic redshifts $z \geqslant 3.06$, such that the NB3640 probes blueward of the Lyman limit. There are also 2 $z>3.06$ QSOs, and 41 LBG photometric candidates without spectroscopic redshifts. The images were taken at a sky position angle of $\theta=180^{\circ}$, with the long axis of the image oriented along the north-south direction. The LRIS-B $4 \mathrm{~K} \times 4 \mathrm{~K} \mathrm{CCD} \mathrm{mosaic}$ detector consists of two $2 \mathrm{~K} \times 4 \mathrm{~K}$ chips with slightly different ( $\sim 20 \%$ ) quantum efficiency at $3640 \AA$, and the pointing center and sky position angle were chosen to maximize the number of $z \geqslant 3.06$ targets falling on the blue side chip with better quantum efficiency. 
Table 1

Description of Observations

\begin{tabular}{lccc}
\hline \hline Filter & Telescope/Instrument & $\begin{array}{c}\text { Seeing FWHM } \\
\left({ }^{\prime \prime}\right)\end{array}$ & $\begin{array}{c}\text { Exposure } \\
(\mathrm{s})\end{array}$ \\
\hline NB3640 & Keck/LRIS-B & 0.80 & 47636 \\
NB4980 & Keck/LRIS-R & 0.80 & 33880 \\
& Subaru/Suprime-Cam & 0.76 & 25800 \\
\hline$B$ & Subaru/Suprime-Cam & 0.76 & 3927 \\
$V$ & Subaru/Suprime-Cam & 0.60 & 3000 \\
$R$ & Subaru/Suprime-Cam & 0.56 & 3000 \\
\hline
\end{tabular}

Individual narrowband exposures were reduced using standard procedures of flatfielding, background subtraction, and removal of cosmic rays. For both NB3640 and NB4980 imaging, flatfields were constructed from de-registered images of the twilight sky. For imaging in each narrowband filter, tasks from the IRAF "images" and "mscred" packages were used to solve for the world coordinate system of each exposure, sample each exposure onto a reference world coordinate system, and shift and combine all individual exposures into a final stacked image. During combination of individual exposures, which ranged in airmass from 1.06 to 1.37 , scaling was applied so that each exposure effectively was observed at the minimum airmass. Furthermore, LRIS-B images were resampled onto the 0.211 LRIS-R pixel scale. Narrowband images were calibrated onto the AB system using observations of spectrophotometric standard stars from the list of Massey et al. (1988). NB3640 and NB4980 AB magnitudes were also corrected for a Galactic extinction of $E(B-V)=0.08$, based on IRAS $100 \mu \mathrm{m}$ cirrus emission maps (Schlegel et al. 1998).

We augmented the LRIS data set with both narrowband and broadband archival imaging data taken with Suprime-Cam (Miyazaki et al. 2002) on the Subaru Telescope. Additional data include narrowband images taken through a filter with central wavelength $4977 \AA$ and FWHM $80 \AA$, i.e., almost identical to the one we used for Keck/LRIS observations, and described previously in Matsuda et al. (2004), Hayashino et al. (2004), and Iwata et al. (2009). These "NB497" images were obtained on 2002 September 8-9 (UT), for a total of $7.2 \mathrm{hr}$ of integration time, and an image quality in the stack of 0.76 FWHM. Broadband $B$ - and $V$-band images were obtained on 2003 September 21 (UT; PI: Hu) with integration times of 1.1 and $0.8 \mathrm{hr}$, respectively, and respective image quality of 0.76 and 0.60 . Finally, we analyze $R$-band imaging from 2001 October 20 (UT; Hu et al. 2004), with an integration time of $0.8 \mathrm{hr}$ and seeing of 0.56 . All Suprime-Cam data were retrieved from the Subaru archive, SMOKA (Baba et al. 2002), and reduced with the SDFRED reduction package (Yagi et al. 2002; Ouchi et al. 2004). Subaru $B, V$-band, and $R$ images were flux calibrated using a combination of spectrophotometric and Landolt (Landolt 1992) standard stars. A summary of both Keck/LRIS and Subaru/Suprime-Cam observations is presented in Table 1.

Given the similarity of the Ly $\alpha$ narrowband filters used for LRIS and Suprime-Cam imaging, in terms of central wavelength and FWHM, we combined NB4980 and NB497 images, scaling them to a common zeropoint, and weighting them according to their respective depths. This combined NB4980 image is $\sim 0.4$ mag deeper than the Subaru-only image presented in Matsuda et al. (2004). The selection of LAEs requires a continuum image probing the same effective wavelength as the narrowband. Since the combination of $B$ and $V$ filters straddles the NB4980 bandpass, a linear combination of the two filters suffices for the matched continuum image. We constructed a $B V$ continuum image by first smoothing the $V$-band image so that the $B$ and $V$ image point-spread functions (PSFs) were matched, and then scaling the PSF-matched images to the same zeropoint. We then executed: $B V=0.44 B+0.56 \mathrm{~V}$, with the weights determined by the relative wavelengths of the broadband and NB4980 filters. We note that the linear combination of $B$ and $V$ images described here differs from that in Matsuda et al. (2004) (i.e., $(2 B+V) / 3)$, and better reflects the relative wavelengths probed by the $B, V$, and NB4980 filters. Finally, we smoothed the $B V$ continuum image so that the PSF matched that of the NB4980 combined image. As described in Section 3.2, selection of LAEs is based on the NB4980 magnitudes and $B V-$ NB4980 colors of objects. However, for examining the spatial distribution of Ly $\alpha$ emission (and absorption), isolated from the local continuum, we also constructed a continuumsubtracted Ly $\alpha$ image, hereafter referred to as $L y A$.

To estimate the noise properties and depth of the images from which photometry was measured (NB3640, LRIS+SuprimeCam NB4980, $B V$-continuum, and $R$ ), we evaluated the counts in apertures distributed randomly in regions free of detected sources. To facilitate comparison with Iwata et al. (2009), we used 1'.2 diameter circular apertures. The standard deviation of the counts in empty apertures yields the associated $1 \sigma$ magnitude limit, from which an estimate of the $3 \sigma$ limiting magnitude can be derived. We find $3 \sigma$ limiting magnitudes of 27.90, 27.90, 28.40, and 27.60, respectively, for the NB3640, combined LRIS+Suprime-Cam NB4980, $B V$-continuum, and $R$ images. Our NB3640 image is therefore roughly 0.6 deeper than the analogous NB359 image presented in Iwata et al. (2009). As described in Section 3.1, this simple estimate of the noise properties of our images does not take into account the full systematic uncertainty associated with source detection and evaluation. Monte Carlo simulations replicating our detection and measurement process with artificial galaxies of known magnitude indicate larger photometric errors than inferred from the stated depths above. All error bars presented in later sections are based on the more conservative Monte Carlo simulations.

Finally, for detailed rest-frame UV (non-ionizing) continuum morphologies of our SSA22a targets, we assembled archival Hubble Space Telescope (HST) Advanced Camera for Surveys (ACS) imaging of the field, taken with the F814W filter. These data include three pointings from the Gemini Deep Deep Survey (Abraham et al. 2007; P.I.D. 9760), with five-orbit exposure times, and three pointings from the program described in Geach et al. (2009; P.I.D. 10405), with three-orbit exposure times. Of the 34 targets with NB3640 detections described in Section 3.4, 25 have deep ACS coverage.

\section{ANALYSIS}

\subsection{Photometric Measurements and Uncertainties}

Source identification and photometry were performed using SExtractor version 2.5.0 (Bertin \& Arnouts 1996). We first smoothed the NB3640 image using a circular Gaussian kernel with $\sigma=1$ pixel $=0$ '.211. We then ran SExtractor in "dualimage mode," using the smoothed image to detect sources though the measurements were performed on the unsmoothed image. We found this method produced superior results in terms of detecting faint sources while not producing overly large apertures, compared to the use of SExtractor's builtin filtering option. Similarly, the NB4980 image was used to 
Table 2

Uncertainties in Simulated Photometry: $R$ and NB3640

\begin{tabular}{lcccc}
\hline \hline Magnitude Range $^{\mathrm{a}}$ & \multicolumn{1}{c}{$\Delta R^{\mathrm{b}}$} & $\sigma_{R}^{\mathrm{c}}$ & $\Delta \mathrm{NB} 3640^{\mathrm{b}}$ & $\sigma_{\mathrm{NB} 3640}^{\mathrm{c}}$ \\
\hline $23.0-23.5$ & 0.00 & 0.05 & -0.01 & 0.03 \\
$23.5-24.0$ & 0.00 & 0.08 & -0.01 & 0.04 \\
$24.0-24.5$ & -0.01 & 0.11 & -0.02 & 0.06 \\
$24.5-25.0$ & -0.01 & 0.16 & -0.03 & 0.10 \\
$25.0-25.5$ & -0.02 & 0.23 & -0.04 & 0.15 \\
$25.5-26.0$ & -0.01 & 0.29 & -0.06 & 0.20 \\
$26.0-26.5$ & 0.01 & 0.37 & -0.06 & 0.29 \\
$26.5-27.0$ & 0.13 & 0.40 & -0.03 & 0.38 \\
$27.0-27.5$ & 0.43 & 0.55 & 0.03 & 0.49 \\
\hline
\end{tabular}

Notes.

a Recovered range of $R$ or NB3640 magnitude for simulated galaxies

$\mathrm{b}$ Average value of the difference between measured and input magnitudes. Significant departures from zero imply systematic biases in the photometry.

c The standard deviation in the difference between measured and input magnitudes.

identify sources and define isophotes for measurements in the $B V$ image for the purpose of identifying LAE candidates (see below).

Due to the details of the radiative transfer and sources of the corresponding photons, any detected Ly $\alpha$ emission and escaping LyC flux will not necessarily be co-spatial with either each other or with the bulk of the rest-frame UV flux in a given galaxy. Therefore, single-image mode was employed for measurements in the NB4980 and $R$ images with no prior used to define apertures, and we report "Kron-like" (i.e., SExtractor MAG_AUTO) total magnitudes for these and the NB3640 images. All reported colors are determined by taking the differences of these magnitudes. This method allows more flexibility (e.g., in avoiding contamination from neighboring sources) and decreases the photometric uncertainty, as compared to using apertures of fixed shape and size large enough to capture all of the flux from the most extended sources. Furthermore, we restrict "detections" to magnitude ranges for which any systematics in the photometry are small compared to the random error (see below).

In addition to the estimates of the depth reached in each image described above (Section 2), we ran simulations of our method in order to obtain a detailed description of the combined systematic and statistical uncertainties in our measurements. We placed one hundred simulated sources of known magnitude into the NB3640 image, the $R$ image, and of known magnitude and color simultaneously into the NB4980 and $B V$ images. The simulated sources were placed at random positions chosen to avoid both real and previously placed simulated sources. SExtractor was then run on these simulated source-added images in the same manner as above (i.e., in single-image mode on $R$ and NB4980 and dual-image mode for detection/measurement on smoothed/ unsmoothed NB3640 and NB4980/ $B V$ ) and magnitudes were recorded for all recovered simulated sources. This process was repeated until a large enough total number (typically $\sim 50,000$ ) of simulated sources had been recovered to populate each bin in magnitude (and color) to a statistically significant degree. The average differences and standard deviations of the recovered from input magnitudes then define any systematics and the uncertainty as a function of magnitude (and color). We list the systematics and uncertainties in NB3640 and $R$ in Table 2, and present those for NB4980 and $B V-\mathrm{NB} 4980$ in Table 3.

\subsection{Ly $\alpha$ Emitter Candidates}

The wavelength of redshifted Ly $\alpha$ falls within our NB4980 filter for sources at $3.054 \lesssim z \lesssim 3.120 .^{4}$ Figure 2 shows isophotal $B V-\mathrm{NB} 4980$ color as a function of total NB4980 magnitude for all sources detected in the NB4980 image. As the isophotes defined in the NB4980 image were used for the $B V$ photometry, source-matching was not an issue. Note that objects identified by SExtractor within Ly $\alpha$ "blobs" (e.g., Steidel et al. 2000) were removed from the catalog. The solid line indicates our formal lower limit in $B V-\mathrm{NB} 4980$ color. For sources bright in NB4980, the limit in color depends primarily on the detection limit $B V=27.5$. At fainter narrowband magnitudes, the color limit is determined empirically from the Monte Carlo simulations described above.

Following Steidel et al. (2000), we identify as LAE candidates sources having $B V-\mathrm{NB} 4980 \geqslant 0.7$. The dotted lines in Figure 2 indicate the $\pm 3 \sigma$ scatter of the main distribution of sources, which is dominated by objects outside of the redshift range $3.054 \lesssim z \lesssim 3.120$. The right-hand axis of Figure 2 shows the Ly $\alpha$ rest-frame equivalent width, $\mathrm{EW}_{0}$, implied by the $B V-\mathrm{NB} 4980$ color for sources in this redshift range. The limit $B V-\mathrm{NB} 4980 \geqslant 0.7$ (corresponding to an observed Ly $\alpha$ equivalent width $\simeq 80 \AA$ ) is above this scatter down to $\mathrm{NB} 4980 \simeq 26$. A total of 110 sources meet the criteria $B V-\mathrm{NB} 4980 \geqslant 0.7$ and $\mathrm{NB} 4980 \leqslant 26$, which we use to define our main LAE candidate sample. For reference, our simulations indicate the uncertainty in color is $\sigma_{B V-\mathrm{NB} 4980} \simeq$ 0.2 for $B V-\mathrm{NB} 4980=0.7$ and $\mathrm{NB} 4980=26$. We also consider sources with $26<\mathrm{NB} 4980 \leqslant 26.5$ with the more conservative color-cut of $B V-\mathrm{NB} 4980 \geqslant 1.2$ as an additional "faint" LAE sample. Twenty sources meet these criteria. As these sources are selected using different criteria, they are excluded from any following calculations using the ensemble of LAEs, unless otherwise noted. The limits defining these sample are shown in Figure 2 with dashed lines.

To test the robustness of the LAE selection technique, we compared the equivalent widths implied by the $B V-\mathrm{NB} 4980$ colors for the 18 LBGs in the field with $3.05 \leqslant z \leqslant 3.12$ (excluding three within Ly $\alpha$ "blobs") to those determined from existing LRIS spectra (Shapley et al. 2003, 2006). The equivalent width values determined from the two methods correlate strongly. The photometrically determined widths are on average larger, which may be due to slit losses in the spectra as the spatial extent of the Ly $\alpha$ emission tends to be larger than that of the rest-frame UV (Hayashino et al. 2004; Steidel et al. 2011). Importantly, the spectra of all five LBGs selected as LAEs exhibit Ly $\alpha$ in emission, and no LBGs with spectroscopic observed equivalent widths greater than $80 \AA$ are missed. Additionally, we verified that none of the 45 galaxies with spectroscopic redshifts $z<3.05$ in the field were selected as LAEs. We also ran SExtractor on the LyA (NB4980-BV difference) image; all of the 130 LAE candidates were recovered as detections.

\subsection{Object Matching}

For each LBG, we compiled the positions and photometric measurements for any SExtractor detection in the NB3640 image within a $2^{\prime \prime}$ (corresponding to $15 \mathrm{kpc}$ at $z=3.09$ ) offset from the center of the $R$-band aperture. For each LAE candidate

\footnotetext{
4 Our NB3640 filter is opaque to non-ionizing UV flux above $z \gtrsim 3.06$ and transparent to only negligible levels of non-ionizing flux at $z \simeq 3.054$.
} 
Table 3

Uncertainties in Simulated Photometry: NB4980 and $B V-\mathrm{NB} 4980$

\begin{tabular}{|c|c|c|c|c|c|}
\hline NB4980 ${ }^{\mathrm{a}}$ & $B V-\mathrm{NB} 4980^{\mathrm{a}}$ & $\Delta \mathrm{NB} 4980^{\mathrm{b}}$ & $\sigma_{\mathrm{NB} 4980^{\mathrm{c}}}$ & $\Delta(B V-\mathrm{NB} 4980)^{\mathrm{b}}$ & $\overline{\sigma_{B V-\mathrm{NB} 3640^{\mathrm{c}}}}$ \\
\hline \multirow[t]{10}{*}{$23.0-23.5 \ldots \ldots . .}$. & $0.0-0.2$ & 0.01 & 0.03 & 0.00 & 0.03 \\
\hline & $0.2-0.4$ & 0.01 & 0.04 & 0.00 & 0.03 \\
\hline & $0.4-0.6$ & 0.01 & 0.04 & 0.00 & 0.03 \\
\hline & $0.6-0.8$ & 0.01 & 0.03 & -0.00 & 0.03 \\
\hline & $0.8-1.0$ & 0.01 & 0.04 & -0.00 & 0.04 \\
\hline & $1.0-1.2$ & 0.01 & 0.04 & -0.00 & 0.05 \\
\hline & $1.2-1.4$ & 0.01 & 0.03 & -0.00 & 0.06 \\
\hline & $1.4-1.6$ & 0.01 & 0.03 & -0.01 & 0.07 \\
\hline & $1.6-1.8$ & 0.01 & 0.04 & -0.01 & 0.09 \\
\hline & $1.8-2.0$ & 0.01 & 0.03 & -0.02 & 0.11 \\
\hline \multirow[t]{10}{*}{$23.5-24.0 \ldots \ldots \ldots$} & $0.0-0.2$ & 0.01 & 0.05 & 0.01 & 0.04 \\
\hline & $0.2-0.4$ & 0.01 & 0.05 & 0.00 & 0.04 \\
\hline & $0.4-0.6$ & 0.02 & 0.05 & 0.00 & 0.04 \\
\hline & $0.6-0.8$ & 0.01 & 0.05 & 0.00 & 0.05 \\
\hline & $0.8-1.0$ & 0.01 & 0.06 & 0.00 & 0.06 \\
\hline & $1.0-1.2$ & 0.01 & 0.05 & -0.01 & 0.07 \\
\hline & $1.2-1.4$ & 0.01 & 0.05 & -0.01 & 0.09 \\
\hline & $1.4-1.6$ & 0.01 & 0.05 & -0.01 & 0.09 \\
\hline & $1.6-1.8$ & 0.01 & 0.05 & -0.03 & 0.13 \\
\hline & $1.8-2.0$ & 0.02 & 0.05 & -0.03 & 0.15 \\
\hline \multirow[t]{10}{*}{$24.0-24.5 \ldots \ldots \ldots$} & $0.0-0.2$ & 0.02 & 0.08 & 0.00 & 0.05 \\
\hline & $0.2-0.4$ & 0.01 & 0.08 & 0.01 & 0.05 \\
\hline & $0.4-0.6$ & 0.02 & 0.09 & 0.01 & 0.06 \\
\hline & $0.6-0.8$ & 0.02 & 0.08 & 0.00 & 0.06 \\
\hline & $0.8-1.0$ & 0.02 & 0.06 & -0.00 & 0.07 \\
\hline & $1.0-1.2$ & 0.02 & 0.07 & -0.01 & 0.09 \\
\hline & $1.2-1.4$ & 0.01 & 0.08 & -0.01 & 0.11 \\
\hline & $1.4-1.6$ & 0.01 & 0.08 & -0.02 & 0.14 \\
\hline & $1.6-1.8$ & 0.02 & 0.08 & -0.04 & 0.18 \\
\hline & $1.8-2.0$ & 0.01 & 0.08 & -0.02 & 0.18 \\
\hline \multirow[t]{10}{*}{$24.5-25.0 \ldots \ldots \ldots$} & $0.0-0.2$ & 0.02 & 0.10 & 0.02 & 0.07 \\
\hline & $0.2-0.4$ & 0.03 & 0.12 & 0.01 & 0.08 \\
\hline & $0.4-0.6$ & 0.03 & 0.12 & 0.01 & 0.08 \\
\hline & $0.6-0.8$ & 0.03 & 0.11 & 0.00 & 0.09 \\
\hline & $0.8-1.0$ & 0.02 & 0.12 & 0.01 & 0.11 \\
\hline & $1.0-1.2$ & 0.02 & 0.11 & -0.02 & 0.14 \\
\hline & $1.2-1.4$ & 0.02 & 0.13 & -0.01 & 0.16 \\
\hline & $1.4-1.6$ & 0.02 & 0.13 & -0.02 & 0.20 \\
\hline & $1.6-1.8$ & 0.03 & 0.11 & -0.04 & 0.21 \\
\hline & $1.8-2.0$ & 0.02 & 0.12 & -0.07 & 0.27 \\
\hline \multirow[t]{10}{*}{$25.0-25.5 \ldots \ldots .}$. & $0.0-0.2$ & 0.04 & 0.15 & 0.03 & 0.10 \\
\hline & $0.2-0.4$ & 0.03 & 0.17 & 0.03 & 0.10 \\
\hline & $0.4-0.6$ & 0.02 & 0.18 & 0.02 & 0.11 \\
\hline & $0.6-0.8$ & 0.02 & 0.16 & 0.01 & 0.13 \\
\hline & $0.8-1.0$ & 0.03 & 0.14 & 0.00 & 0.14 \\
\hline & $1.0-1.2$ & 0.03 & 0.16 & -0.01 & 0.19 \\
\hline & $1.2-1.4$ & 0.01 & 0.18 & -0.05 & 0.24 \\
\hline & $1.4-1.6$ & 0.03 & 0.16 & -0.05 & 0.26 \\
\hline & $1.6-1.8$ & 0.02 & 0.17 & -0.08 & 0.32 \\
\hline & $1.8-2.0$ & 0.03 & 0.16 & -0.10 & 0.38 \\
\hline \multirow[t]{10}{*}{$25.5-26.0 \ldots \ldots \ldots$} & $0.0-0.2$ & 0.04 & 0.22 & 0.05 & 0.13 \\
\hline & $0.2-0.4$ & 0.06 & 0.21 & 0.03 & 0.13 \\
\hline & $0.4-0.6$ & 0.04 & 0.22 & 0.04 & 0.15 \\
\hline & $0.6-0.8$ & 0.05 & 0.23 & 0.01 & 0.19 \\
\hline & $0.8-1.0$ & 0.01 & 0.25 & -0.01 & 0.22 \\
\hline & $1.0-1.2$ & 0.02 & 0.24 & -0.03 & 0.24 \\
\hline & $1.2-1.4$ & 0.05 & 0.24 & -0.05 & 0.28 \\
\hline & $1.4-1.6$ & 0.03 & 0.24 & -0.15 & 0.44 \\
\hline & $1.6-1.8$ & 0.02 & 0.20 & -0.11 & 0.45 \\
\hline & $1.8-2.0$ & 0.05 & 0.21 & -0.13 & 0.43 \\
\hline \multirow[t]{10}{*}{$26.0-26.5 \ldots \ldots .}$. & $0.0-0.2$ & 0.07 & 0.26 & 0.04 & 0.15 \\
\hline & $0.2-0.4$ & 0.10 & 0.28 & 0.05 & 0.17 \\
\hline & $0.4-0.6$ & 0.07 & 0.26 & 0.04 & 0.20 \\
\hline & $0.6-0.8$ & 0.08 & 0.28 & 0.03 & 0.22 \\
\hline & $0.8-1.0$ & 0.05 & 0.28 & 0.00 & 0.27 \\
\hline & $1.0-1.2$ & 0.07 & 0.33 & -0.02 & 0.33 \\
\hline & $1.2-1.4$ & 0.08 & 0.29 & -0.07 & 0.37 \\
\hline & $1.4-1.6$ & 0.04 & 0.26 & -0.14 & 0.48 \\
\hline & $1.6-1.8$ & 0.06 & 0.27 & -0.12 & 0.48 \\
\hline & $1.8-2.0$ & 0.06 & 0.28 & -0.12 & 0.49 \\
\hline
\end{tabular}

Notes.

${ }^{a}$ Recovered magnitude or color range of simulated galaxies.

${ }^{b}$ Average value of the difference between measured and input magnitudes or colors. Significant departures from zero imply systematic biases in the photometry.

$\mathrm{c}$ The standard deviation in the difference between measured and input magnitudes or colors. 


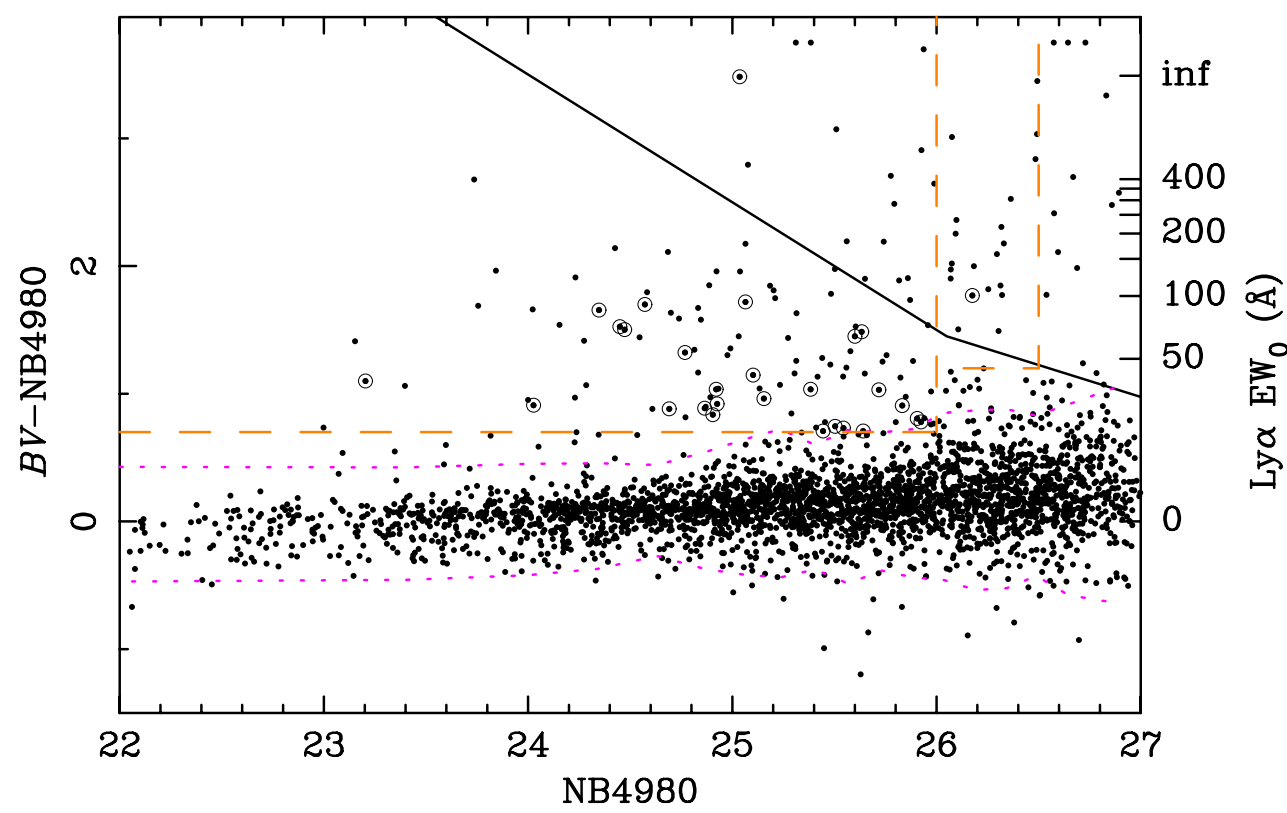

Figure 2. Color-magnitude diagram for sources detected in the NB4980 image. Right axis indicates the rest-frame Ly $\alpha$ equivalent width (assuming $z=3.09$ ) implied by the $B V-\mathrm{NB} 4980$ color for sources at $3.054 \lesssim z \leqslant 3.12$. Isophotes for $B V-\mathrm{NB} 4980$ colors are defined in the NB4980 image, while NB4980 magnitudes are "Kronlike" (i.e., SExtractor MAG_AUTO) total magnitudes. The solid curve indicates our formal lower-limit threshold in $B V-$ NB4980, though sources with measured values above this limit are plotted with their measured values, excepting those with measured colors $B V-\mathrm{NB} 4980>3.75$ which are plotted as $B V-\mathrm{NB} 4980=3.75$. Dotted lines indicate $\pm 3 \sigma$ scatter in the data and dashed lines indicate our limits defining the LAE candidate sample. Circled points indicate sources with NB3640 detections.

(A color version of this figure is available in the online journal.)

(hereafter, LAE), we retained any detections in the NB3640 and $R$ image within $2^{\prime \prime}$ of the NB4980 aperture center. We visually inspected each matched source in all available bands, including the deep $B V$ and the high image quality $R$, and removed any obvious false matches corresponding to neighboring sources. In rare cases, the deblending produced by SExtractor was unsatisfactory and the photometry was recomputed using the IRAF task phot.

Six of the 26 LBGs were detected in our NB3640 image. We retain the NB3640 detection near C16 despite the large (1'.9) offset, as there are no neighbors detected in any of the other bands coincident with the position of the NB3640 flux. Of the 110 main sample LAEs, 27 were detected in NB3640 and 72 were detected in $R$ with $R \leqslant 27$, which we adopt as our formal detection limit based on our simulations discussed in Section 3.1. As with C16, a small number of detections near LAEs having fairly large $\left(\sim 1^{\prime \prime} 5-2^{\prime \prime}\right)$ offsets are retained as the LAE is the closest source detected in any of the other bands. We account for the possibility of mismatched NB3640 detections in Section 3.5. One of the 20 faint sample LAEs was also detected in the NB3640 image, and two detected in the $R$-band image with $R \leqslant 27$.

\subsection{LBGs and LAEs with NB3640 Detection}

Table 4 lists the coordinates, redshifts based on Ly $\alpha$ emission and interstellar absorption (when available), $R$ and NB3640 magnitudes, and spatial offsets of the $R$ and NB3640 emission centroids $\left(\Delta_{R}\right)$ for the six LBGs with NB3640 detections. Of the two LBGs reported by Shapley et al. (2006) to exhibit LyC flux in their spectra, we detect one (C49), consistent with the results of Iwata et al. (2009). The 28 (27 main and one faint sample) LAEs with NB3640 detections are marked with circles in Figure 2, and the coordinates, photometry, $\mathrm{Ly} \alpha \mathrm{EW}_{0}$, and $\Delta_{R}$ and $L y A-\mathrm{NB} 3640\left(\Delta_{L y A}\right)$ spatial offsets are presented in Table 5 . We also note that five of the 26 LBGs in our sample qualify as LAEs by our criteria, though none of these are detected in NB3640. Information for LBGs and LAEs with no NB3640 detection is given in the Appendix.

Figure 3 displays the smoothed NB3640, R, and HST/ACSF814W images for the six LBGs with NB3640 detections. Figures 4(a)-(e) display the smoothed NB3640, LyA, $R$, and HST/ACS-F814W images for the 28 (main and faint sample) LAEs with NB3640 detections. The contours in these figures represent NB3640 flux levels corresponding to 28.81, 28.06, and $27.62 \mathrm{mag} \mathrm{arcsec}^{-2}(1 \sigma, 2 \sigma$, and $3 \sigma$ pixel-to-pixel fluctuations in the smoothed image).

We also investigated the average $R$-band and NB3640 flux of various subsamples of LBGs and LAEs by stacking postage stamp images of the individual galaxies. Regions containing flux from sources obvious neighbors were removed using masks created from the SExtractor "segmentation" images. Both the $R$-band and NB3640 postage stamps were centered on the $R$ detection for LBGs and on the NB4980 detection for LAEs and, after the rejection of masked pixels, averaged to create each stack. No significant NB3640 flux was detected in either the stack of the 20 LBGs (down to a $2 \sigma$ limiting magnitude of 28.14 ) or of the 83 LAEs (down to a $2 \sigma$ limiting magnitude of 28.91) lacking individual NB3640 detections. ${ }^{5}$ The results from the photometry on stacked subsamples are reported in Table 6 .

As can be seen in Figures 3 and 4(a)-(e) (as well as Tables 4 and 5), the NB3640, Ly $\alpha$, and $R$-band fluxes are not precisely cospatial in many of our sources. Spatial offsets between detected ionizing and non-ionizing UV continuum fluxes (i.e., significant values of $\Delta_{R}$ ), have been previous noted (Iwata et al. 2009; Inoue et al. 2011). The values of $\Delta_{R}$ in our LAE sample

\footnotetext{
5 Although we did not test the NB3640 filter in the lab for the possibility of a "red leak," the strong detection limits in the stacks indicate that there is no such leak at a level that could affect our results.
} 
Table 4

Photometry for LBGs with NB3640 Detections

\begin{tabular}{|c|c|c|c|c|c|c|c|c|c|}
\hline ID & $\begin{array}{l}\text { R.A. } \\
\text { (J2000) }\end{array}$ & $\begin{array}{l}\text { Decl. } \\
\text { (J2000) }\end{array}$ & $z_{\mathrm{em}}{ }^{\mathrm{a}}$ & $z_{\mathrm{abs}}{ }^{\mathrm{b}}$ & $R$ & NB3640 & $\Delta_{R}^{\mathrm{c}}$ & $\frac{F_{\mathrm{UV}}}{F_{\mathrm{LyC}}}$ obs & $\frac{F_{\mathrm{UV}}}{F_{\mathrm{LyC}}}$ cor \\
\hline MD46 & $22: 17: 27.28$ & 0:18:09.7 & 3.091 & 3.080 & 23.49 & 25.22 & 1.0 & $4.9 \pm 0.7$ & $2.4 \pm 1.0$ \\
\hline C16 & $22: 17: 31.95$ & $0: 13: 16.3$ & $\ldots$ & 3.0651 & 23.62 & 26.43 & 1.9 & $13.3 \pm 4.0$ & $6.8 \pm 3.3$ \\
\hline C49 & $22: 17: 19.81$ & $0: 18: 18.8$ & 3.1629 & 3.1492 & 23.81 & 26.84 & 0.4 & $16.4 \pm 6.1$ & $5.9 \pm 4.1$ \\
\hline D17 & $22: 17: 18.86$ & $0: 18: 17.0$ & 3.0898 & 3.0697 & 24.29 & 27.00 & 0.9 & $12.2 \pm 5.1$ & $5.9 \pm 3.5$ \\
\hline aug96M16 & $22: 17: 30.86$ & $0: 13: 10.8$ & 3.298 & 3.285 & 24.47 & 25.23 & 0.7 & $2.0 \pm 0.4$ & $0.2 \pm 0.4$ \\
\hline MD32 & $22: 17: 23.70$ & 0:16:01.6 & 3.102 & $\ldots$ & 25.14 & 25.51 & 0.4 & $1.4 \pm 0.4$ & $0.6 \pm 0.3$ \\
\hline
\end{tabular}

Notes.

${ }^{\text {a }}$ Ly $\alpha$ emission redshift.

${ }^{b}$ Interstellar absorption redshift.

${ }^{c}$ Spatial offset between the centroids of $R$-band and NB3640 emission.

${ }^{\mathrm{d}}$ Observed ratio and uncertainty in non-ionizing UV and LyC emission, inferred from the NB3640- $R$ color. This value has not been corrected for either contamination by foreground sources or IGM absorption.

${ }^{\mathrm{e}}$ Ratio of non-ionizing UV continuum and LyC emission, corrected for both foreground contamination and IGM absorption.

Table 5

Photometry for LAEs with NB3640 Detections

\begin{tabular}{|c|c|c|c|c|c|c|c|c|c|c|c|}
\hline ID & $\begin{array}{l}\text { R.A. } \\
\text { (J2000) }\end{array}$ & $\begin{array}{c}\text { Decl. } \\
\text { (J2000) }\end{array}$ & 4980 & $B V-\mathrm{NB} 4980$ & $\begin{array}{c}\mathrm{EW}_{0}{ }^{\mathrm{a}} \\
(\AA ̊)\end{array}$ & $R$ & NB3640 & $\Delta_{R}^{\mathrm{b}}$ & $\Delta_{L y A} \mathrm{~b}$ & $\frac{F_{\mathrm{UV}}}{F_{\mathrm{LyC}}}{ }_{\mathrm{obs}}{ }^{\mathrm{c}}$ & $\frac{F_{\mathrm{UVV}}}{F_{\mathrm{LyC}}}$ cor \\
\hline 003 & $22: 17: 24.79$ & $0: 17: 17.4$ & 23.20 & 1.10 & 39 & 24.42 & 24.74 & $0 ! 3$ & $0{ }^{\prime \prime} 6$ & $1.3 \pm 0.1$ & $0.6 \pm 0.3$ \\
\hline 010 & $22: 17: 20.38$ & 0:18:04.2 & 24.03 & 0.91 & 28 & 25.77 & 26.74 & $0 ! 3$ & 0.3 & $2.4 \pm 1.1$ & $1.2 \pm 0.7$ \\
\hline 016 & $22: 17: 35.61$ & 0:18:00.2 & 24.35 & 1.66 & 86 & 26.24 & 26.91 & 0.9 & 0.8 & $1.8 \pm 0.9$ & $0.9 \pm 0.6$ \\
\hline 018 & $22: 17: 39.01$ & $0: 17: 26.4$ & 24.45 & 1.53 & 72 & 26.25 & 25.69 & 0.1 & 1.0 & $0.6 \pm 0.2$ & $0.3 \pm 0.2$ \\
\hline 019 & $22: 17: 26.15$ & $0: 13: 20.1$ & 24.47 & 1.50 & 70 & 25.70 & 26.24 & 0.4 & 0.9 & $1.6 \pm 0.6$ & $0.8 \pm 0.4$ \\
\hline 021 & $22: 17: 18.77$ & $0: 15: 18.1$ & 24.57 & 1.70 & 92 & $>27$ & 27.17 & $1 " .3$ & 1..4 & $<1.8$ & $<0.9$ \\
\hline 025 & $22: 17: 36.74$ & $0: 16: 28.8$ & 24.69 & 0.88 & 27 & 25.54 & 25.85 & 0.3 & 1 1.2 2 & $1.3 \pm 0.4$ & $0.6 \pm 0.3$ \\
\hline 028 & $22: 17: 31.80$ & $0: 17: 17.9$ & 24.77 & 1.32 & 54 & 25.50 & 26.71 & $0 ! 3$ & 0.9 & $3.1 \pm 1.3$ & $1.5 \pm 0.9$ \\
\hline 034 & $22: 17: 23.41$ & $0: 16: 35.4$ & 24.86 & 0.89 & 27 & 25.42 & 25.76 & 0,5 & $0^{\prime \prime} 0$ & $1.4 \pm 0.4$ & $0.7 \pm 0.3$ \\
\hline 038 & $22: 17: 34.77$ & $0: 15: 41.3$ & 24.90 & 0.84 & 25 & 26.17 & 25.82 & $0^{\prime \prime} 1$ & 0.7 & $0.7 \pm 0.3$ & $0.3 \pm 0.2$ \\
\hline 039 & $22: 17: 24.08$ & $0: 11: 31.7$ & 24.92 & 1.03 & 35 & 26.48 & 26.77 & 0.4 & 0.8 & $1.3 \pm 0.7$ & $0.6 \pm 0.4$ \\
\hline 041 & $22: 17: 24.54$ & $0: 15: 06.7$ & 24.93 & 0.92 & 29 & 25.97 & 25.94 & 0.4 & 0.5 & $1.0 \pm 0.4$ & $0.5 \pm 0.3$ \\
\hline 046 & $22: 17: 21.47$ & $0: 14: 54.6$ & 25.04 & $>2.54$ & $>277$ & $>27$ & 26.43 & 1 1". 8 & 1.. 8 & $<0.8$ & $<0.4$ \\
\hline 048 & $22: 17: 27.37$ & $0: 16: 51.5$ & 25.06 & 1.72 & 94 & 26.73 & 26.00 & $2 ! 2$ & $1 . .7$ & $0.5 \pm 0.2$ & $0.2 \pm 0.2$ \\
\hline 051 & $22: 17: 33.72$ & 0:15:04.9 & 25.10 & 1.15 & 41 & 26.26 & 27.21 & $0 ! 3$ & 0.7 & $2.4 \pm 1.3$ & $1.2 \pm 0.8$ \\
\hline 053 & $22: 17: 34.70$ & $0: 16: 33.4$ & 25.15 & 0.96 & 31 & 26.53 & 26.98 & $00^{\prime} 6$ & 0.9 & $1.5 \pm 0.8$ & $0.7 \pm 0.5$ \\
\hline 064 & $22: 17: 35.42$ & $0: 12: 14.6$ & 25.38 & 1.04 & 35 & 26.05 & 26.74 & 0.1 & 1 .. 0 & $1.9 \pm 0.9$ & $0.9 \pm 0.6$ \\
\hline 069 & $22: 17: 18.96$ & $0: 11: 12.0$ & 25.44 & 0.71 & 19 & 24.62 & 27.22 & $0 ! 2$ & 0.9 & $10.9 \pm 5.1$ & $5.3 \pm 3.3$ \\
\hline 074 & $22: 17: 36.47$ & $0: 12: 54.8$ & 25.50 & 0.75 & 21 & 26.16 & 25.52 & $0 ! .1$ & 0.7 & $0.6 \pm 0.2$ & $0.3 \pm 0.1$ \\
\hline 077 & $22: 17: 37.95$ & $0: 11: 01.3$ & 25.54 & 0.73 & 20 & 26.01 & 26.36 & $0 ! 2$ & 1 ." 1 & $1.4 \pm 0.6$ & $0.7 \pm 0.4$ \\
\hline 081 & $22: 17: 29.22$ & $0: 14: 48.7$ & 25.60 & 1.45 & 65 & $>27$ & 26.79 & $0^{\prime \prime} 6^{\mathrm{e}}$ & 0.7 & $<1.2$ & $<0.6$ \\
\hline 083 & $22: 17: 28.46$ & 0:12:08.9 & 25.63 & 1.48 & 68 & 26.46 & 26.84 & 0.4 & $0^{\prime} .6$ & $1.4 \pm 0.7$ & $0.7 \pm 0.5$ \\
\hline 084 & $22: 17: 19.90$ & $0: 15: 14.9$ & 25.64 & 0.71 & 20 & $>27$ & 26.50 & 0,1 & 0.8 & $<0.9$ & $<0.4$ \\
\hline 087 & $22: 17: 37.07$ & $0: 13: 21.5$ & 25.72 & 1.03 & 35 & $>27$ & 27.26 & $0^{\prime} 1^{\mathrm{e}}$ & 1.. 6 & $<2.0$ & $<1.0$ \\
\hline 096 & $22: 17: 38.93$ & $0: 11: 37.4$ & 25.83 & 0.91 & 28 & 26.64 & 26.45 & $0{ }^{\prime \prime} 6$ & $0^{\prime} .6$ & $0.8 \pm 0.4$ & $0.4 \pm 0.3$ \\
\hline 101 & $22: 17: 25.33$ & $0: 17: 22.5$ & 25.90 & 0.81 & 24 & 26.89 & 26.58 & $0 ! 2$ & $1 . .4$ & $0.8 \pm 0.4$ & $0.4 \pm 0.2$ \\
\hline 102 & $22: 17: 24.00$ & $0: 16: 27.6$ & 25.92 & 0.78 & 22 & 25.91 & 26.05 & 0.1 & 0.2 & $1.1 \pm 0.4$ & $0.6 \pm 0.3$ \\
\hline 118 & $22: 17: 35.28$ & $0: 10: 59.9$ & 26.18 & $>1.39$ & $>243$ & 26.20 & 26.11 & 0.4 & 1 1.. 4 & $0.9 \pm 0.4$ & $0.4 \pm 0.3$ \\
\hline
\end{tabular}

Notes.

${ }^{a}$ Ly $\alpha$ rest equivalent width estimated from $B V-\mathrm{NB} 4980$ color.

${ }^{\mathrm{b}}$ Spatial offset between the centroids of $R$ or Ly $\alpha$ and NB3640 emission.

${ }^{c}$ Observed ratio and uncertainty in non-ionizing UV and LyC emission, inferred from the NB3640- $R$ color. This value has not been corrected for either contamination by foreground sources or IGM absorption.

${ }^{\mathrm{d}}$ Ratio of non-ionizing UV continuum and LyC emission, corrected for both foreground contamination and IGM absorption.

${ }^{\mathrm{e}}$ Offset determined from the centroid of $B V$ detection.

are relatively small $\left(\lesssim 0{ }^{\prime \prime} 6\right)$, however, compared to the values of $\Delta_{L y A}$ and $L y A-R$ offsets $\left(\Delta_{L y A, R}\right)$. The median values for main sample LAEs with NB3640 detections are $\Delta_{R}=0.32$, $\Delta_{L y A}=00^{\prime \prime} 82$ and $\Delta_{L y A, R}=0{ }^{\prime \prime} 58$. We show the individual values of $\Delta_{L y A}$ versus $\Delta_{R}$, as well as $\Delta_{R}$ versus NB3640 magnitude, in Figure 5.

\subsection{The Source of the NB3640 Detections}

The detections in the NB3640 image coincident with the locations of $z \gtrsim 3.06$ LBGs and LAEs are likely due to some combination of (1) photons of wavelengths shortward of the Lyman limit escaping from the galaxies and being 


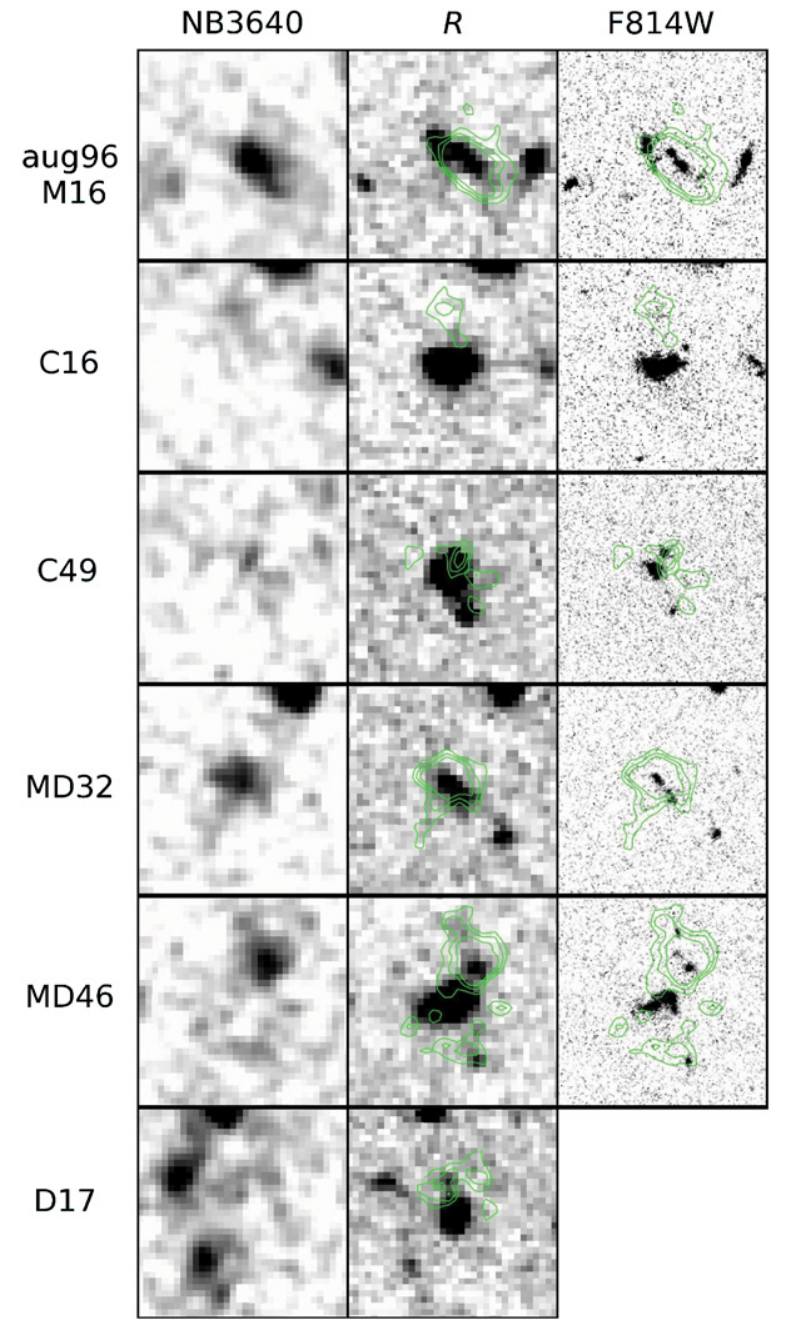

Figure 3. LBGs with $z>3.06$ detected in NB3640. Images are centered on the $R$-band centroid and span $7^{\prime \prime} \times 7^{\prime \prime}$. The NB3640 filter (image shown here after smoothing) samples the rest-frame $\mathrm{LyC}$, while the $R$ and $\mathrm{F} 814 \mathrm{~W}$ filters sample the rest-frame non-ionizing UV continuum.

(A color version of this figure is available in the online journal.) redshifted above the Lyman break before the neutral phase of the IGM is able to absorb them and (2) non-ionizing flux from lower-redshift galaxies randomly coincident on the sky with the high $-z$ systems. In principle, the high spatial resolution of the HST/ACS imaging available for many of our sources with NB3640 detections could help distinguish between the scenarios. However, galaxies observed in the rest-frame UV wavelengths often appear clumpy (Lotz et al. 2006; Law et al. 2007; Peter et al. 2007)—i.e., as distinct regions significantly brighter than the regions surrounding them-particularly at high redshift. Thus, even with the resolution afforded by HST/ACS imaging, it is not generally possible to distinguish in individual systems between clumpy emission from a single redshift $(z \approx 3)$ and multiple sources at different redshifts.

It is possible to address the issue of foreground contamination in a statistical manner, however. The positions of any foreground objects will be uncorrelated with that of the $z \gtrsim 3.06$ sources, and their surface density can be predicted using, e.g., $U$-band number counts in the ultradeep VLT/VIMOS catalogue (Nonino et al. 2009). We predict the surface density of such sources, $\rho_{S}$, from the Nonino et al. (2009) number counts by interpolating the values in Table 1 of Vanzella et al. (2010a) for our magnitude range $24.5<U<27.25$. To define the positions of the 26 LBGs, we use the centroids of the $R$-band detections, which have confirmed spectroscopic redshifts $z>3.06$. For the 110 LAEs, we first use the centroids of the detections in the LyA image (although, see below), which are very likely to be in the redshift range $3.054 \lesssim z \lesssim 3.120$. In Figure 6, we show the radial surface density of all NB3640 detections surrounding the $z \gtrsim 3.06$ sources, in bins of radial offset (i.e., $\Delta_{R}$ for the LBGs and $\Delta_{L y A}$ for the LAEs). The surface density is defined as the number of detections divided by $\pi\left(r_{\max }^{2}-r_{\min }^{2}\right) N$ where $r_{\min }$ and $r_{\max }$ are the minimum and maximum radii of the bin and $N$ is the number of $z \gtrsim 3.06$ sources, 26 for the LBGs and 110 for the LAEs. The left-hand panel shows the distribution for the LBG sample, while the bottom right panel shows the distribution for the LAEs. The shaded portion of the histograms represent detections that we have identified as clearly belonging to a neighboring source other than the target LBG/LAE (and

Table 6

Photometry in Stacked Images

\begin{tabular}{|c|c|c|c|c|c|c|c|}
\hline Sample & Num. & $R$ & $\mathrm{NB} 3640^{\mathrm{a}}$ & $\sigma_{\mathrm{NB} 3640-R}^{\mathrm{phot}}$ & $\sigma_{\mathrm{NB} 3640-R}^{\mathrm{sample}}$ & NB3640- $R$ & $\left\langle F_{\mathrm{UV}} / F_{\mathrm{LyC}}\right\rangle_{\text {obs }}{ }^{\mathrm{d}}$ \\
\hline LBGs & 26 & 24.23 & 27.94 & 0.41 & 0.91 & $3.71 \pm 1.00$ & $30.5_{-18.3}^{+46.1}$ \\
\hline LBGs, non-detect & 20 & 24.32 & $>28.14$ & $\ldots$ & $\ldots$ & $>3.82$ & $>33.7$ \\
\hline LBGs, detect & 6 & 23.98 & 25.81 & 0.13 & 0.23 & $1.83 \pm 0.26$ & $5.4_{-1.2}^{+1.5}$ \\
\hline LAEs & 110 & 26.14 & 27.49 & 0.14 & 0.32 & $1.35 \pm 0.35$ & $3.5_{-1.0}^{+1.3}$ \\
\hline LAEs, non-detect & 83 & 26.19 & $>28.91$ & $\ldots$ & $\ldots$ & $>2.72$ & $>12.2$ \\
\hline LAEs, detect & 27 & 26.04 & 26.22 & 0.14 & 0.57 & $0.18 \pm 0.59$ & $1.2_{-0.5}^{+1.9}$ \\
\hline LAEs, $R<25$ & 8 & 24.44 & 27.16 & 0.36 & 0.94 & $2.72 \pm 1.01$ & $12.2_{-7.4}^{+18.8}$ \\
\hline LAEs, $25<R<26$ & 23 & 25.64 & 26.69 & 0.15 & 0.26 & $1.05 \pm 0.30$ & $2.6_{-0.6}^{+0.8}$ \\
\hline LAEs, $26<R<27$ & 43 & 26.68 & 27.59 & 0.27 & 0.53 & $0.91 \pm 0.59$ & $2.3_{-1.0}^{+1.7}$ \\
\hline LAEs, $R>27$ & 36 & 27.53 & 28.41 & 0.67 & 0.84 & $0.88 \pm 1.07$ & $2.2_{-1.4}^{+3.8}$ \\
\hline Faint LAEs & 20 & 27.93 & $>28.13$ & $\ldots$ & $\ldots$ & $>0.20$ & $>1.2$ \\
\hline
\end{tabular}

Notes.

a Limits, when quoted, are $2 \sigma$.

b Photometric color uncertainty determined from stacks of randomly sampled blank regions of the $R$ and NB3640 images.

c Sample color uncertainty determined by bootstrap resampling of the subsample.

${ }^{\mathrm{d}}$ Observed ratio and uncertainty in non-ionizing UV and LyC emission, inferred from the NB3640- $R$ color of subsample stacks. This value has not been corrected for either contamination by foreground sources or IGM absorption. 


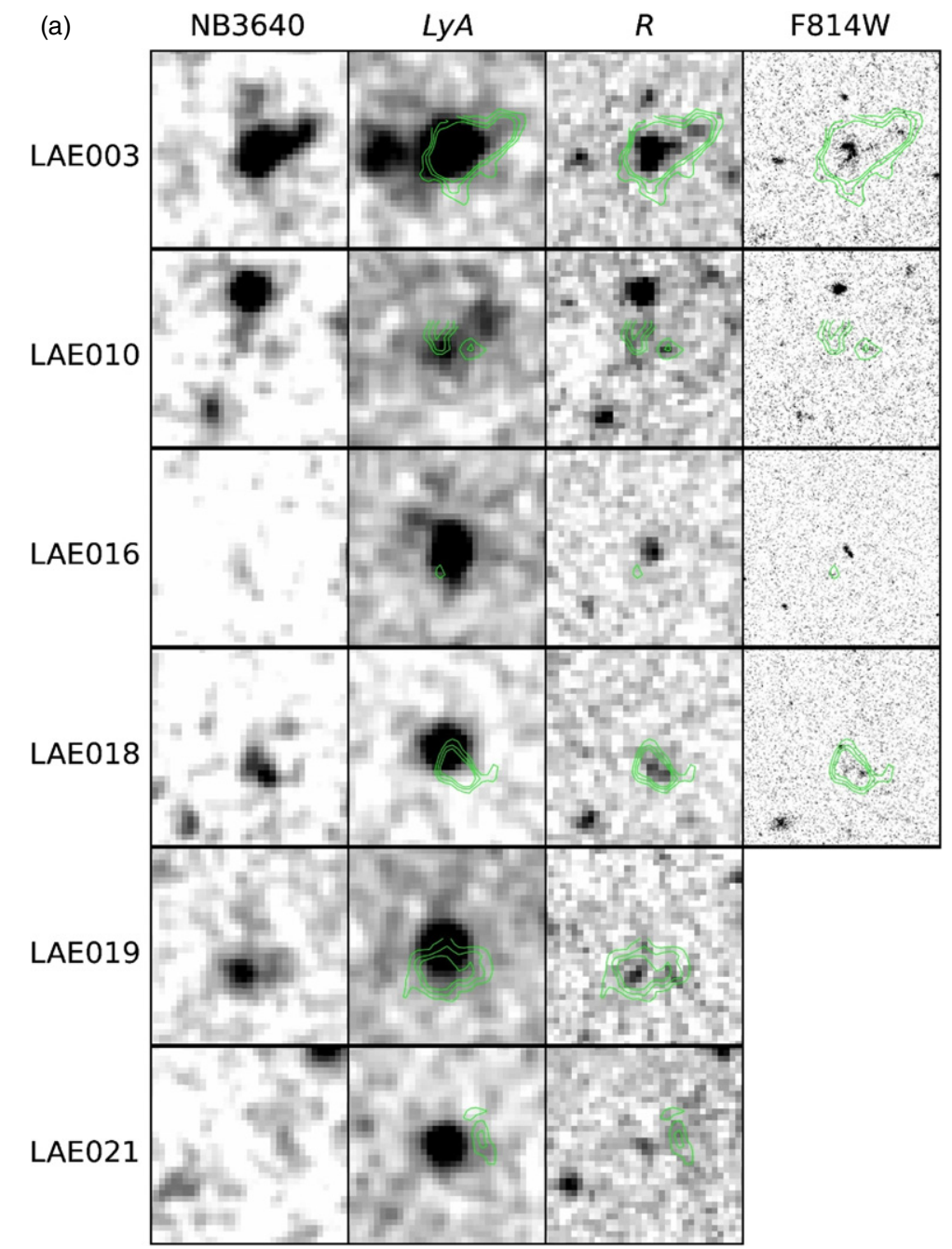

Figure 4. (a)-(e) As in Figure 3, but for LAEs with NB3640 detections. Also shown is the LyA (i.e., $B V$-continuum-subtracted NB4980, see the text) image. Images are centered on the $L y A$ detection and span $7^{\prime \prime} \times 7^{\prime \prime}$.

(A color version of this figure is available in the online journal.)

therefore do not appear in Tables 4 and 5). The dashed and dotted lines indicate the predicted surface density of interlopers, $\rho_{S}$, and the $1 \sigma$ uncertainty in the prediction, respectively. At large offset, the distributions are comprised entirely of obvious neighbors, flat and consistent with $\rho_{S}$. This consistency indicates that any possible difference between the $U$-band number counts and the surface density of NB3640 sources in our field (e.g., from the differences in filters, cosmic variance, etc.) can be ignored for our purposes. While disentangling true detections from interlopers becomes more difficult at smaller offsets, there is clearly a significant excess of detections above the average foreground level, indicating that many of the retained NB3640 detections with offsets $\lesssim 1^{\prime \prime}$ must be physically related to the redshift $z \geqslant 3.06$ sources. The magnitude of this excess over that predicted by the $U$-band number counts represents the predicted number of uncontaminated $\mathrm{LyC}$ detections, which we quantify below.

We use $\rho_{S}$ to compute the full (radially dependent) probability distribution for the number of interlopers in our samples. Following Vanzella et al. (2010a), the probability that a given LBG/LAE has a random foreground source with an offset in the range $r_{\min }: r_{\max }$ is then

$$
p_{r}=\pi\left(r_{\max }^{2}-r_{\min }^{2}\right) \times \rho_{S} .
$$

The probability that $K$ of the $N=131$ sources have a contaminant centered within the annulus is

$$
f_{r}(K)=\left(\begin{array}{l}
N \\
K
\end{array}\right) p_{r}^{K}\left(1-p_{r}\right)^{(N-K)} .
$$

The expectation value for the number of contaminated LBGs/ LAEs is

$$
\left\langle n_{r}\right\rangle=\sum_{K} K f_{r}(K)
$$

Note that dividing $\left\langle n_{r}\right\rangle$ by the areas corresponding to each bin in Figure 6, one recovers the surface density indicated by the dashed line.

Having determined $f_{r}(K)$ for bins of offset, we can use Monte Carlo simulations to make a robust prediction of the number of uncontaminated NB3640 detections. In each bin, we randomly select a number of interlopers, $n_{\text {inter }}$, from $f_{r}(K)$. We then subtract the number of previously rejected sources in the bin, $n_{\text {rej }}$ (i.e., those corresponding to obvious neighbors, and indicated by the hatched histogram in Figure 6). If $N=n_{\text {inter }}-n_{\text {rej }}>0$, we randomly reject $N$ of the retained detections in the given bin. We repeated the simulation 1000 times to determine the average and $1 \sigma$ deviation of uncontaminated NB3640 detections. The results of our simulation indicate that $3.2 \pm 1.2$ of 


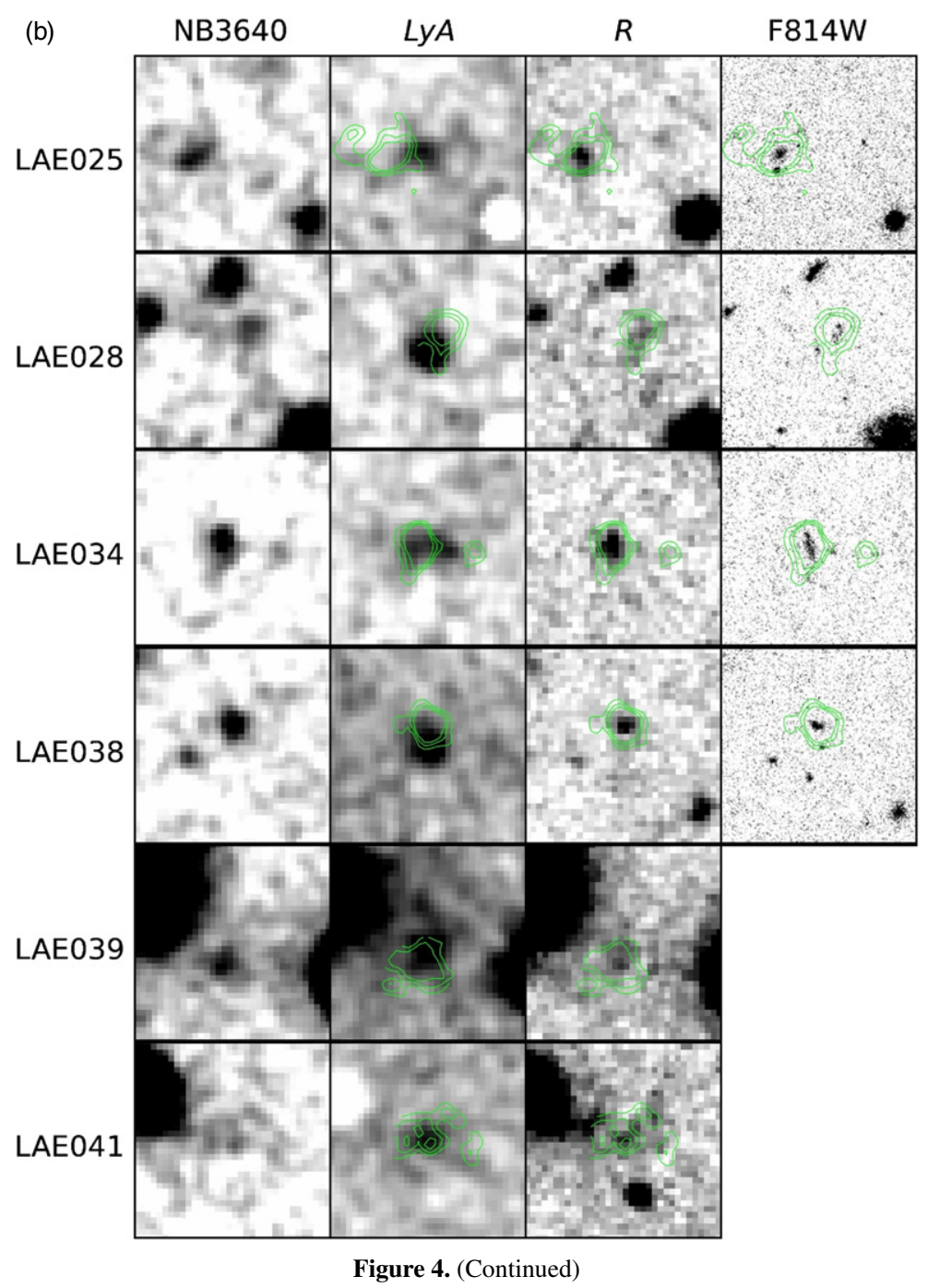

the 26 LBGs and $18.0 \pm 3.2$ of the 110 main sample LAEs have NB3640 detections that are uncontaminated by foreground sources. The two samples are not independent, as they share 5 sources; considering the sample as a whole, our simulation determines that $21.6 \pm 3.8$ of the 131 sources have uncontaminated NB3640 detections. These same simulations can also be used to contamination-correct the ensemble average NB3640 magnitudes determined from the stacked images (Table 6) or by summing the individual magnitudes assuming zero flux from the non-detections (see Section 4.4).

As discussed in Section 3.4, detections in the NB3640 images associated with LAEs are less spatially consistent with the detected Ly $\alpha$ emission than with the rest-frame UV continuum emission. Thus, it will be more suitable to define the LAE positions using the centroids of the $R$-band detections (i.e., use $\Delta_{R}$ in place of $\Delta_{L y A}$ ) for the purpose of estimating the contamination, unless significant numbers of the NB3640 detections that we have associated with $z \simeq 3.09$ LAEs are interlopers and the corresponding $R$-band detection is dominated by the interloper. However, as we concluded above that the majority of such detections are not contaminated, the $\Delta_{R}$ offsets should lead to more realistic predictions of the number of uncontaminated sources. The upper right panel of Figure 6 shows the radial surface density of detections using $\Delta_{R}$ offsets for the 78 LAEs for which an $R$-band centroid was measurable, $\Delta_{B V}$ offsets for 9 LAEs detected in the
$B V$ image but not $R$, and $\Delta_{\mathrm{NB} 4980}$ offsets for the remaining 23 LAEs. Using these offsets, our Monte Carlo simulations predict that $20.8 \pm 2.7$ of the 110 main sample LAEs and $24.5 \pm 3.0$ of the 131 combined (LBG+LAE) sample sources are uncontaminated.

Finally, we consider the possibility that the LAEs with NB3640 detections having small NB3640 $R$ offsets are not at $z \simeq 3.09$, but rather, e.g., [O II] emitters at $z \simeq 0.34$. In this scenario, the NB3640 filter would sample the restframe UV flux and NB4980- $B V$ would be a measure [O II] emission, both of which should closely trace regions of active star formation, while $R$ would correspond to the rest-frame optical. The observed NB3640 flux would then be more spatially correlated with NB4980- $B V$ compared to $R$. As can be seen in Figure 5, however, the opposite is the case.

\section{RESULTS}

One of the primary goals of this work is to estimate the comoving emissivity of ionizing radiation that escapes from starforming galaxies at $z \sim 3$. Ideally, this estimate would entail deep, rest-frame LyC imaging of several large, independent fields to determine the $\lambda \simeq 900 \AA$ luminosity function (LF); however, such an experiment would be prohibitively expensive in terms of observing time. Here, we have taken advantage of the large number of galaxies at $z \geqslant 3.06$ in the SSA22a field, 


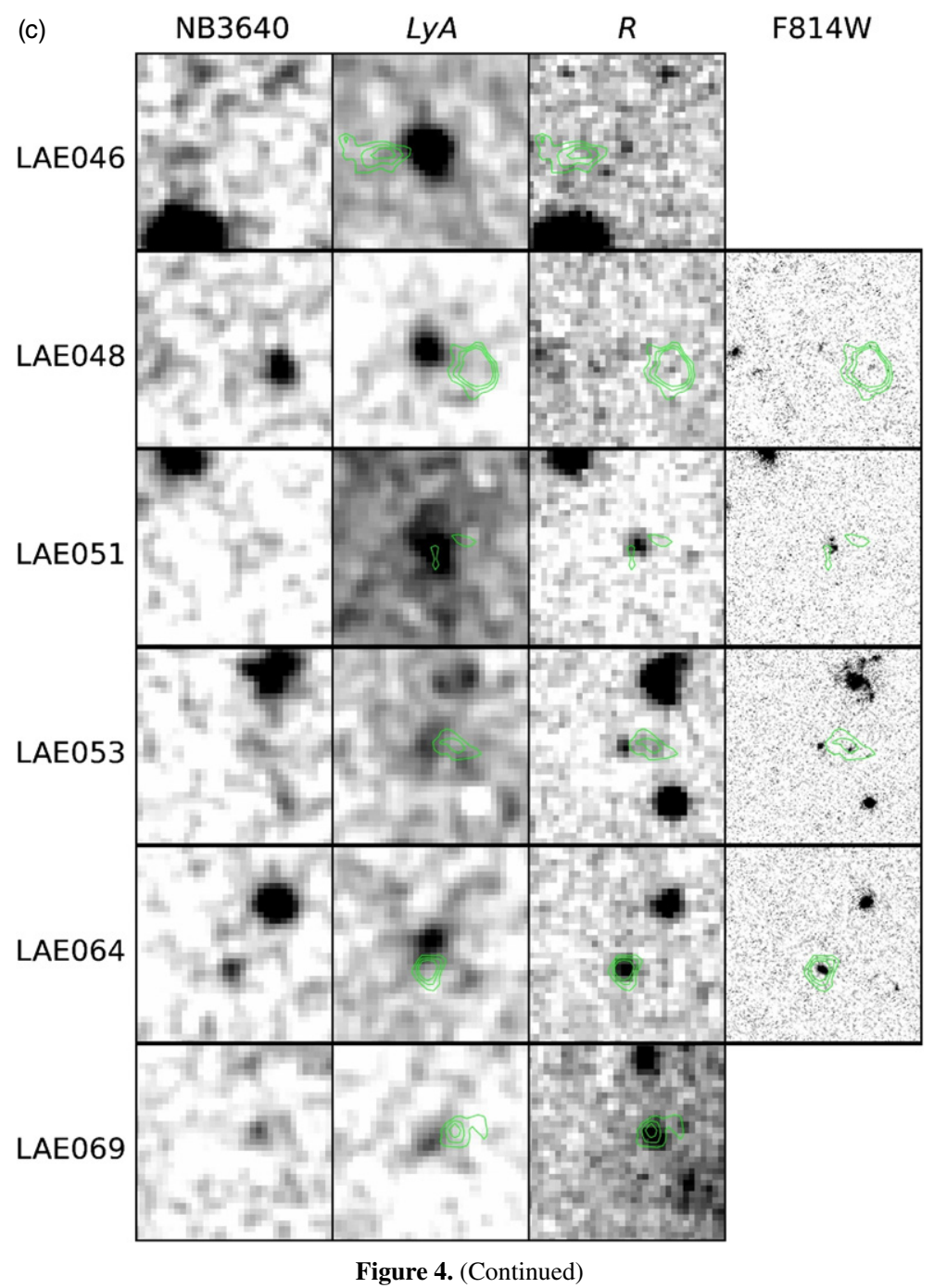

which contains a known protocluster at $z=3.09$, to measure the emergent UV flux escaping from a large sample of LBGs and LAEs both redward and blueward of the Lyman limit. The resulting ratios of non-ionizing to ionizing flux densities allow us to use the previously measured global non-ionizing UV emissivity at $z \sim 3$ to determine the overall contribution to the ionizing UV background at these redshifts from galaxies represented by those in our subsamples.

\subsection{Observed UV-to-Lyman-continuum Flux Density Ratios}

The Suprime-Cam $R$ filter has an effective wavelength of $\sim 1600 \AA$ and FWHM of $\sim 300 \AA$ in the rest frame of our LBG and LAE samples. This is a useful spectral range for determining UV-to-LyC flux density ratios, as the LF at $\lambda \simeq 1700 \AA$ has been well studied at $z \sim 3$ (e.g., Reddy et al. 2008). We show the NB3640-R colors of our sources as a function of $R$ in Figure 7. Also shown are the colors of stacks (Section 3.4) of all LBGs, all LAEs, LBGs and LAEs having no individual NB3640 detection, and subsamples LAEs in bins of $R$. Sources with NB3640 detections in objects brighter than $R \simeq 25$, while few (five LBGs and two LAEs), span a large range NB3640- $R$ color $(\simeq 0-3)$. While the apparent trend in color with $R$ is in part a result of the NB3640 detection limit (indicated by the dotted line in Figure 7) and the increased photometric uncertainty for fainter systems (e.g., $\sigma_{\mathrm{NB} 3640-R} \gtrsim 0.5$ for $R \simeq 26.5, \mathrm{NB} 3640-R \simeq 0$; see Table 2), photometry on the stacks of LBGs and LAEs in bins of $R$ indicates a significant difference in the average NB3640-R color of systems brighter and fainter than $R \simeq 25$.

The observed UV-to-LyC flux density ratios can be computed directly from the values of NB3640 and $R$. We present these ratios in Tables 4 and 5 . Our value for $\mathrm{C} 49, F_{\mathrm{UV}} / F_{\mathrm{LyC}}=$ $16.6 \pm 6.1$, is consistent with that derived from the LRIS spectrum by Shapley et al. (2006), $F_{\mathrm{UV}} / F_{\mathrm{LyC}}=12.7 \pm 1.8$. However, unlike Shapley et al. (2006) we do not detect D3 in NB3640, consistent with the findings of Iwata et al. (2009). The average ratios for the ensembles of LBGs and LAEs are also of interest. These can be computed either from the photometry on the stacked images or from the individual $R$ and NB3640 measurements. Motivated by the non-detection of NB3640 flux in the stacks of individually non-detected sources, we assume zero flux for the non-detections in NB3640 when computing the ensemble ratios from individual measurements. ${ }^{6}$ Doing so, we find values of $\left\langle F_{\mathrm{UV}} / F_{\mathrm{LyC}}\right\rangle_{\mathrm{obs}}=17.8_{-6.6}^{+10.4}$ and $4.2_{-1.0}^{+1.2}$ for the ensembles of LBGs and main sample LAEs, respectively. Considering only sources with NB3640 detections, we find $\left\langle F_{\mathrm{UV}} / F_{\mathrm{LyC}}\right\rangle_{\mathrm{obs}}^{\mathrm{det}}=5.2_{-1.4}^{+1.8}$ for LBGs and $1.3 \pm 0.3$ for LAEs. In all cases, the errors are dominated by the sample variance

\footnotetext{
6 For ease we also assume zero flux in $R$ for LAEs with no $R$-band detection. This assumption has negligible effect on our results.
} 


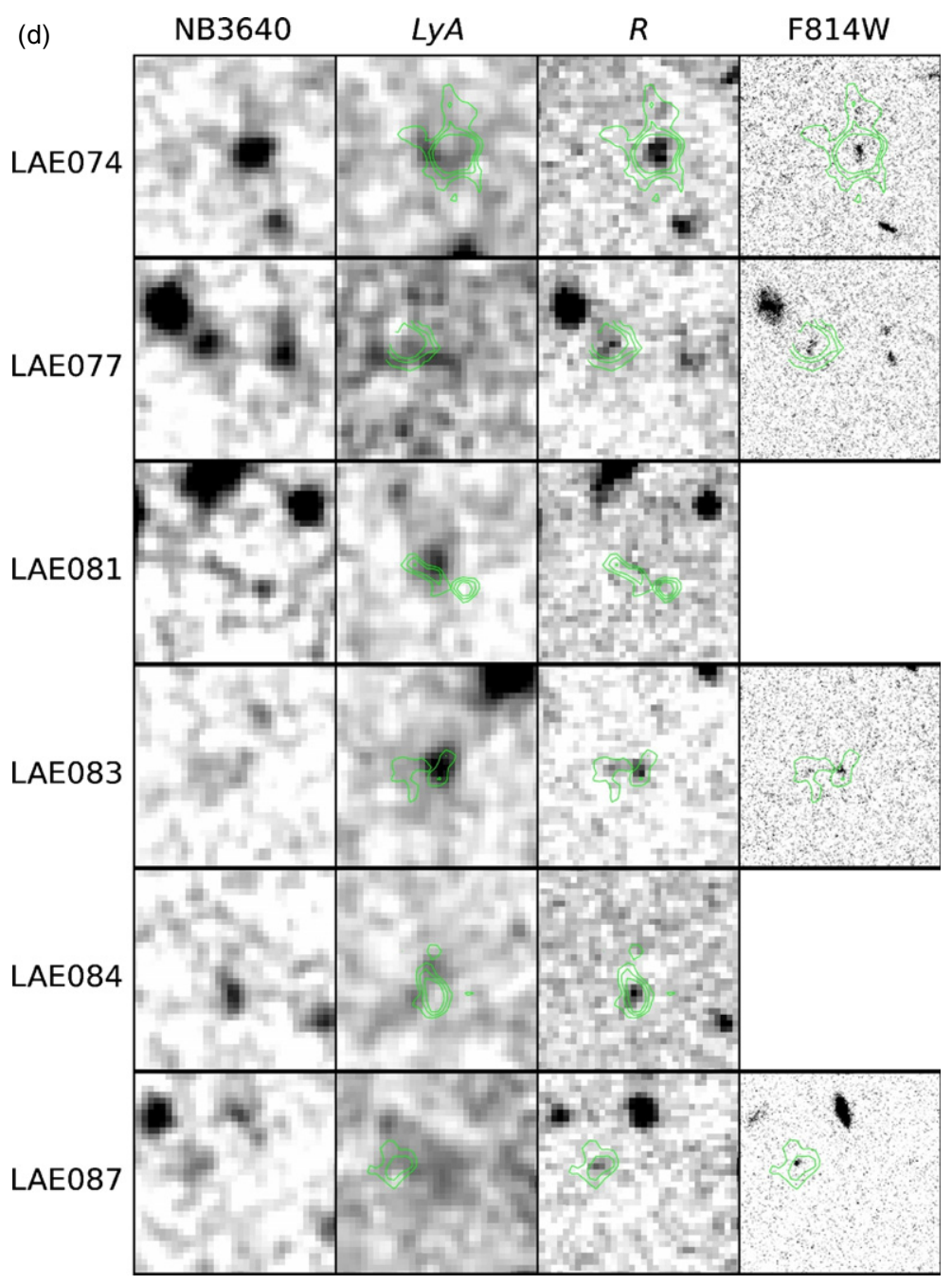

Figure 4. (Continued)

which is determined using bootstrap resampling. The ensemble results are summarized in Table 7. The ratios determined from the stacked data, which we report in Table 6, are consistent with the above values but with larger uncertainties. For the sources with non-detections, we find limits of $\left\langle F_{\mathrm{UV}} / F_{\mathrm{LyC}}\right\rangle_{\mathrm{obs}}^{\text {non-det }}>33.7$ and $>12.2$ for the LBGs and LAEs respectively.

\subsection{IGM Absorption of Lyman-continuum Flux}

Any LyC flux that escapes from the galaxies in our samples is diminished by absorption from the neutral phase of the IGM. In order to model IGM opacity, we use Monte Carlo simulations of intergalactic absorption to generate large (500) samples of random sightlines to the redshifts of our target galaxies. Our simulations are a modified version of those presented in Shapley et al. (2006).

Absorbers are drawn at random from column-density and redshift distributions consistent with recent determinations in the literature (Inoue \& Iwata 2008; Faucher-Giguère et al. 2008; Songaila \& Cowie 2010) for both the lower-column-density Ly $\alpha$ forest, and higher-column-density Lyman limit systems (LLSs) and Damped Ly $\alpha$ systems (DLAs). Of particular relevance for estimating the attenuation just below $912 \AA$ are the statistics for LLSs $\left(N_{\mathrm{HI}} \geqslant 10^{17.2}\right)$. For these systems, we assume a power-law distribution in column-density, proportional to $N_{\mathrm{HI}}^{-1.3}$ (Inoue \& Iwata 2008; Prochaska et al. 2010), redshift evolution proportional to $(1+z)^{2.04}$ (Songaila \& Cowie 2010), with a total number per unit redshift at $z \sim 3$ of $d N / d z \approx 2$ (Songaila \& Cowie 2010; Stengler-Larrea et al. 1995; Steidel 1992). Randomly generated absorbers are then applied to the unabsorbed continuum of each model sightline until the total number of absorbers is equal to a random Poisson realization of the integral of the absorber column-density and redshift distributions over the relevant ranges in column density $\left(N_{\mathrm{HI}}=\right.$ $\left.10^{12}-10^{22} \mathrm{~cm}^{-2}\right)$ and redshift $\left(z=1.7-z_{\text {source }}\right.$, where $z_{\text {source }}$ is the redshift of the target galaxy). The simulations predict a mean free path to ionizing radiation at $z \sim 3$ (as estimated from the redshift at which $F_{\text {cont }} / F_{912}=\exp (-1)$, following Prochaska et al. 2009), which is consistent with recent determinations in the literature of $\lambda_{\text {mfp }} \sim 72-85$ proper Mpc (Faucher-Giguère et al. 2008; Prochaska et al. 2009; Songaila \& Cowie 2010). Using the relation given by Songaila \& Cowie (2010), we adopt a value of $\lambda_{\mathrm{mfp}}=75.6 \mathrm{Mpc}$ at $z=3.1$ for the analysis in Section 4.4.

For each target redshift, the resulting sample of 500 model sightlines is analyzed to obtain the median and standard deviation of the attenuation factor within the NB3640 filter. This quantity differs from the one presented in Shapley et al. (2006), which corresponds to the attenuation factor in the fixed restframe bandpass of 880-910 A. In Shapley et al. (2006), a uniform transmission function was also assumed across this fixed rest-frame bandpass. Here we estimate the attenuation in a fixed 


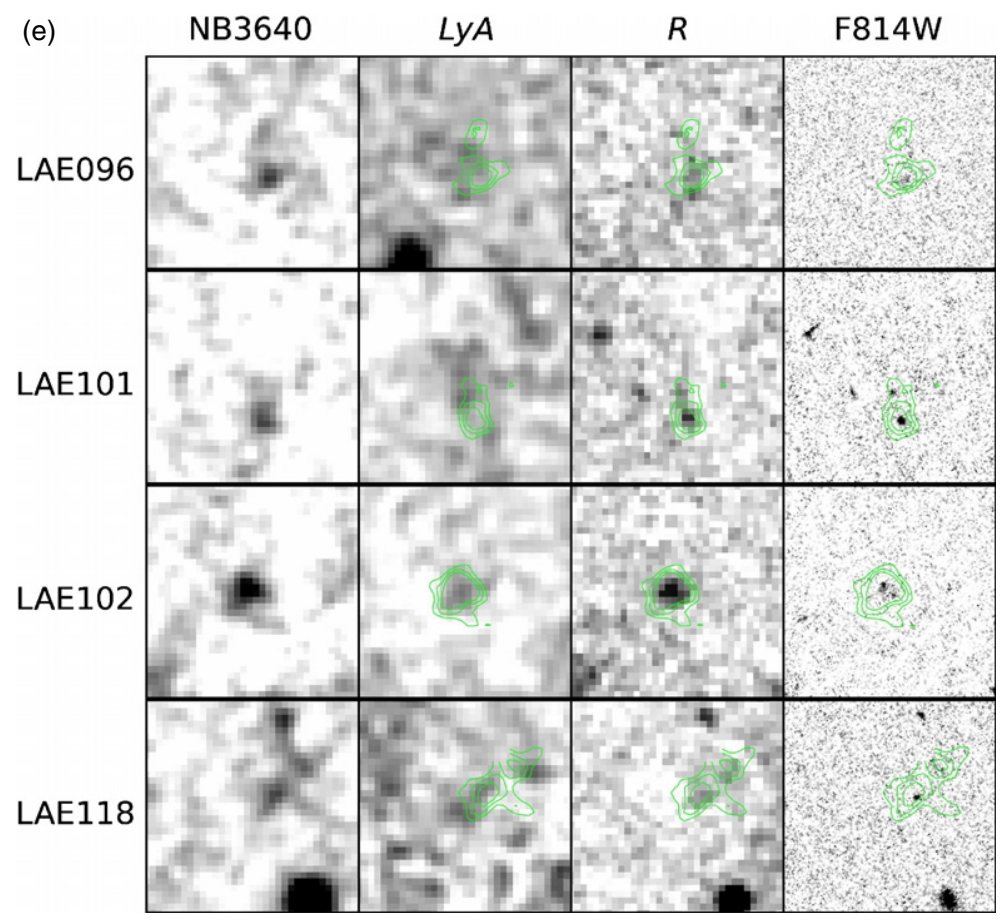

Figure 4. (Continued)
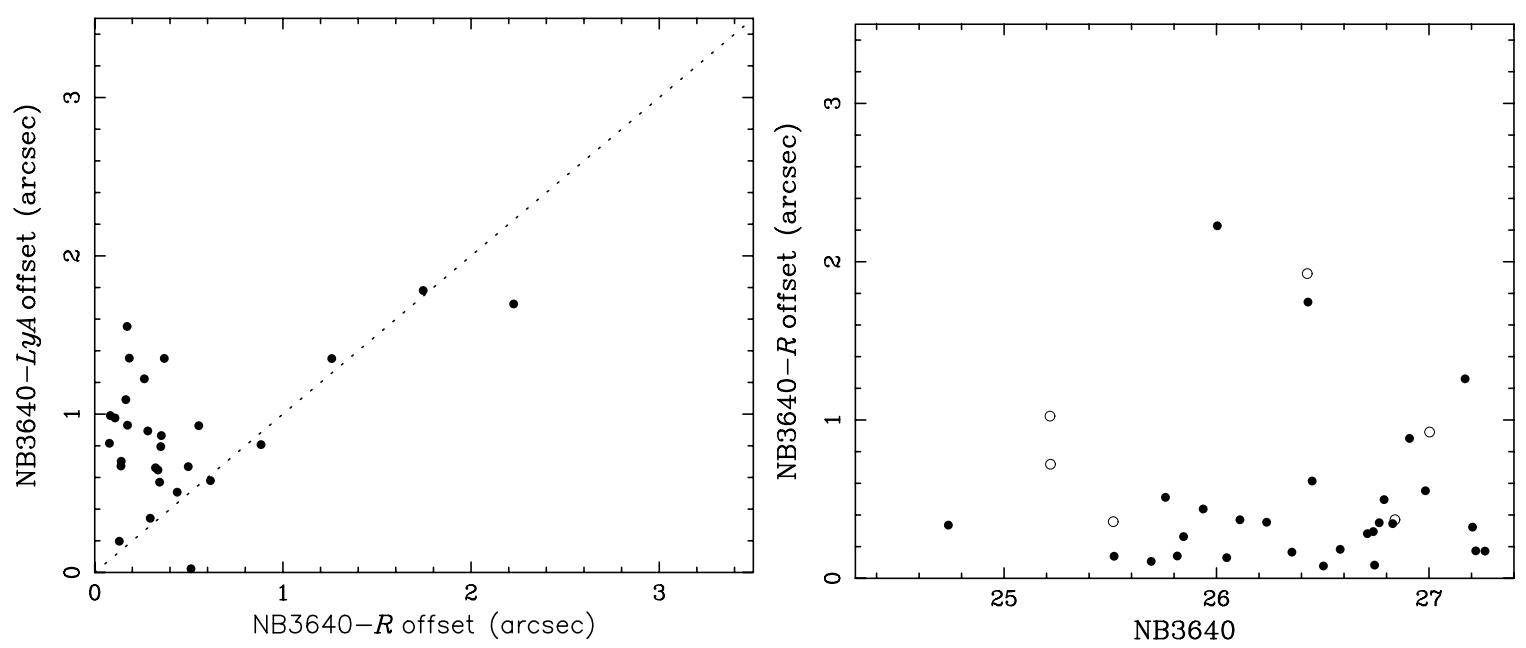

Figure 5. Left: comparison of the spatial offset between detections in the NB3640 and LyA images with those between NB3640 and $R$ for our LAE sample. The smaller NB3640- $R$ offsets relative to NB3640-LyA strongly favors the sources being LAE emitters at $z \simeq 3.09$ over, e.g., [O II] emitters at lower redshift (see the text). Right: NB3640-R spatial offsets vs. NB3640 magnitude. Open points represent LBGs while solid points represent LAEs.

observed frame bandpass, and also take into account the shape of the NB3640 filter. For higher redshifts, the attenuation within the NB3640 filter is stronger not only due to the evolving physical properties of the Ly $\alpha$ forest, but also the fact that the NB3640 filter probes bluer rest-frame wavelengths sensitive to photons that have traveled a longer path length from the emitting galaxy.

For the six LBGs with NB3640 detections, the median and standard deviation of the 500 attenuation factors determined for the appropriate redshift are used to correct the observed UV-toLyC flux density ratios. We assume $z=3.09$ for each LAE. We use the average redshift of the LBG sample, $\langle z\rangle=3.10$, and of the subsample with NB3640 detections, $\langle z\rangle=3.13$, to correct the subsample ratios. In Figure 8, we show the distribution of attenuation factors for our $z=3.09$ simulation as well as the distributions for the LBG and LAE ensemble average attenuation factors. When computing the uncertainties in the median attenuations for LBG and LAE subsamples, we assume the sightlines to the individual sources are uncorrelated. The corrected flux density ratios are presented in Tables 4 and 5 for individual sources, and in Table 7 for ensembles.

\subsection{The Escape Fraction of Ionizing Photons}

The UV-to-LyC flux density ratios determined in our samples of LBGs and LAEs can be used to estimate the fraction of LyC photons that escape from galaxies into the IGM, $f_{\mathrm{esc}}^{\mathrm{LyC}}$. However, we must first correct the ensemble average flux density ratios for contamination (Section 3.5) and IGM absorption (Section 4.2). In each realization of the Monte Carlo simulations described in Section 3.5, we set the NB3640 fluxes to zero for sources selected as interlopers by the simulation. We retain all measured $R$-band fluxes, which is an appropriate approximation if the 
Table 7

Average UV-to-LyC Flux Density Ratios

\begin{tabular}{|c|c|c|c|c|}
\hline \multirow[t]{2}{*}{ Correction } & \multicolumn{2}{|c|}{ LBGs } & \multicolumn{2}{|c|}{ LAEs } \\
\hline & $\langle\mathrm{NB} 3640\rangle-\langle R\rangle^{\mathrm{a}}$ & $\left\langle F_{\mathrm{UV}} / F_{\mathrm{LyC}}\right\rangle^{\mathrm{b}}$ & $\langle\mathrm{NB} 3640\rangle-\langle R\rangle^{\mathrm{a}}$ & $\left\langle F_{\mathrm{UV}} / F_{\mathrm{LyC}}\right\rangle^{\mathrm{b}}$ \\
\hline \multicolumn{5}{|c|}{ Full ensembles } \\
\hline None & $3.13 \pm 0.50$ & $17.8_{-66}^{+10.4}$ & $1.56 \pm 0.28$ & $4.2_{-10}^{+1.2}$ \\
\hline Contamination $^{\mathrm{c}}$ & $3.68 \pm 0.69$ & $29.6_{-13.9}^{+26.3}$ & $1.82 \pm 0.36$ & $5.4_{-1.5}^{+2.1}$ \\
\hline 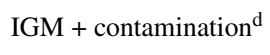 & $2.64 \pm 0.70$ & $11.3_{-5.4}^{+10.3}$ & $0.86 \pm 0.36$ & $2.2_{-0.6}^{+0.9}$ \\
\hline \multicolumn{5}{|c|}{ Sources with NB3640 detections only } \\
\hline None & $1.78 \pm 0.33$ & $5.2_{-1.4}^{+1.8}$ & $0.25 \pm 0.25$ & $1.3 \pm 0.3$ \\
\hline Contamination $^{\mathrm{c}}$ & $1.65 \pm 0.51$ & $4.6_{-1.7}^{+2.7}$ & $0.28 \pm 0.30$ & $1.3_{-0.3}^{+0.4}$ \\
\hline IGM + contamination $^{\mathrm{d}}$ & $0.45 \pm 0.59$ & $1.5_{-0.6}^{+1.1}$ & $-0.69 \pm 0.32$ & $0.5_{-0.1}^{+0.2}$ \\
\hline
\end{tabular}

Notes.

a Color determined from average NB3640 and $R$-band fluxes. Uncertainties include individual flux and sample uncertainties.

${ }^{\mathrm{b}}$ Ratio and uncertainty in non-ionizing UV and LyC emission inferred from $\langle\mathrm{NB} 3640\rangle-\langle R\rangle$ color.

${ }^{\mathrm{c}}$ Color and flux density ratio after statistically correcting sample for foreground contamination of NB3640 fluxes.

${ }^{\mathrm{d}}$ Color and flux density ratio after correcting sample for foreground contamination and IGM absorption of NB3640 fluxes.

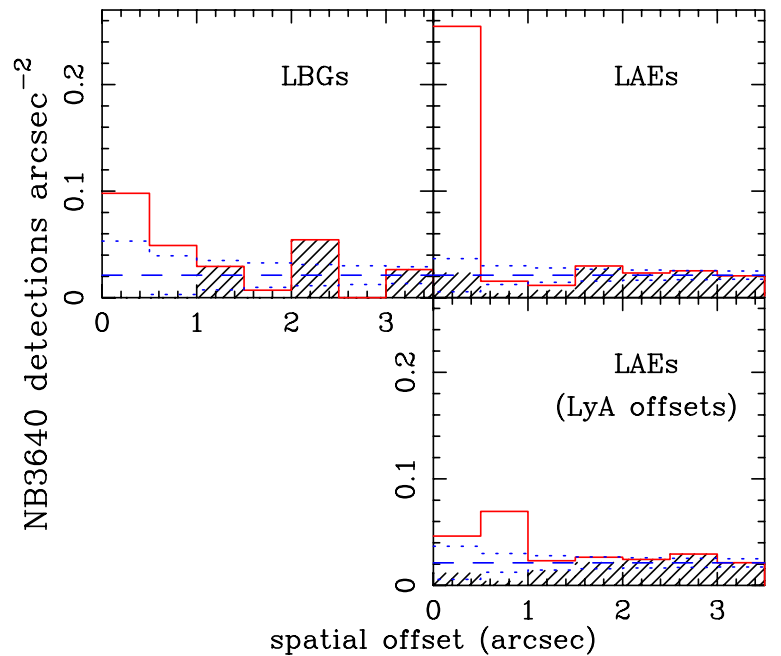

Figure 6. Radial surface density of NB3640 detections around galaxies in our LBG and LAE samples. The solid histograms include all sources identified by SExtractor; the subset of these sources associated with obvious neighbors (which we have excluded from our catalog, see Section 3.3) are represented by the hatched region. The dashed lines indicate the global surface density of sources in our NB3640 magnitude range and thus represents the expected level of contamination, while the dotted lines represent the expected $1 \sigma$ scatter in the contamination. The excess surface density at low offsets indicates that several of our low offset LBG NB3640 detections and the bulk of the low offset LAE NB3640 detections are physically associated with the $z \simeq 3.09$ sources and not random foreground interlopers. The top panels use the displacement of the LyC centroid from the that of the $R$-band detection (i.e., $\Delta_{R}$ ) for LBGs and LAEs with $R$-band detections, the displacements from the $B V$ centroid $\left(\Delta_{B V}\right)$ for LAEs detected in $B V$ but not $R$, and from the NB4980 $\left(\Delta_{\mathrm{NB} 4980}\right)$ detection for the remaining LAEs. The bottom panel uses displacements from the $L y A$ detections $\left(\Delta_{L y A}\right)$ of LAEs. The excess surface density is contained within a larger range of offsets in this panel, as the $\Delta_{R}$ and $\Delta_{B V}$ values tend to be smaller than the $\Delta_{L y A}$ values in our LAE sample (see Figure 5).

(A color version of this figure is available in the online journal.)

interlopers are significantly bluer in observed NB640- $R$ relative to the $z \sim 3$ source, and in any case is the most conservative approximation. We then apply the appropriate IGM correction (Section 4.2) to the average flux density ratios determined from the contamination-corrected samples. We present the raw and corrected colors and flux density ratios for the ensembles and subsamples with NB3640 detections in Table 7. Errors include sample variance computed by first randomly reassigning individual magnitudes based on the measured magnitude and error, assuming Gaussian magnitude uncertainties determined from our photometric simulations (Section 3.1), and then bootstrap resampling the data set. After applying both corrections, we find $\left\langle F_{\mathrm{UV}} / F_{\mathrm{LyC}}\right\rangle_{\text {corr }}^{\mathrm{LBG}}=11.3_{-5.4}^{+10.3}$ for our sample of LBGs. Our sample of LAEs has on average much bluer LyC-UV colors, with a corrected flux density ratio of $\left\langle F_{\mathrm{UV}} / F_{\mathrm{LyC}}\right\rangle_{\text {corr }}^{\mathrm{LAE}}=2.2_{-0.6}^{+0.9}$. The corrected ratios represent the average relative flux density in the vicinity of $z \simeq 3$ galaxies.

The absolute LyC escape fraction can be inferred from the relative escape fraction as $f_{\mathrm{esc}}^{\mathrm{LyC}}=f_{\mathrm{esc}}^{\mathrm{rel}} \times f_{\mathrm{esc}}^{\mathrm{UV}}$, where $f_{\mathrm{esc}}^{\mathrm{UV}}$ is the escape fraction of non-ionizing UV photons and $f_{\text {esc }}^{\text {rel }}$ is determined from the corrected flux density ratio:

$$
f_{\mathrm{esc}}^{\mathrm{rel}}=\frac{\left(L_{\mathrm{UV}} / L_{\mathrm{LyC}}\right)_{\text {intr }}}{\left(F_{\mathrm{UV}} / F_{\mathrm{LyC}}\right)_{\mathrm{corr}}},
$$

and thus

$$
f_{\mathrm{esc}}^{\mathrm{LyC}}=\left(F_{\mathrm{UV}} / F_{\mathrm{LyC}}\right)_{\mathrm{corr}}^{-1} \times\left(L_{\mathrm{UV}} / L_{\mathrm{LyC}}\right)_{\mathrm{intr}} \times f_{\mathrm{esc}}^{\mathrm{UV}},
$$

where $\left(L_{\mathrm{UV}} / L_{\mathrm{LyC}}\right)_{\text {intr }}$ is the (unknown) intrinsic ratio of UVto-LyC luminosity densities produced in star-forming regions. Reddy et al. (2008) report an average UV escape fraction of $f_{\mathrm{esc}}^{\mathrm{UV}} \sim 20 \%-25 \%$ in $z \sim 3$ LBGs. While direct measurements of $\left(L_{\mathrm{UV}} / L_{\mathrm{LyC}}\right)_{\text {intr }}$ are lacking, spectral synthesis models predict values of $\sim 6$ for reasonable assumption of stellar population ages, metallicities, and initial mass functions (IMFs; Siana et al. 2007). We adopt these numbers as our fiducial values, resulting in expressions for the relative and absolute escape fractions: $f_{\mathrm{esc}}^{\mathrm{rel}}=6.0 \times\left(F_{\mathrm{UV}} / F_{\mathrm{LyC}}\right)_{\mathrm{corr}}^{-1}$ and $f_{\mathrm{esc}}^{\mathrm{LyC}}=0.225 \times f_{\mathrm{esc}}^{\mathrm{rel}}$. We caution, however, that sources with escaping LyC flux may also have larger UV escape fractions. In any case, both $f_{\mathrm{esc}}^{\mathrm{UV}}$ and $\left(L_{\mathrm{UV}} / L_{\mathrm{LyC}}\right)_{\text {intr }}$ are highly uncertain and likely vary from object to object. Also note that our fiducial value for $\left(L_{\mathrm{UV}} / L_{\mathrm{LyC}}\right)_{\text {intr }}$ differs from that used by Steidel et al. (2001) and Shapley et al. (2006), who adopt a value of three.

Most of the 20 LBGs with non-detections in NB3640 have only weak individual upper limits on the relative escape fraction, ranging from $f_{\text {esc }}^{\text {rel }} \lesssim 0.5$ to $\sim$ unity. The $(2 \sigma)$ upper limit 


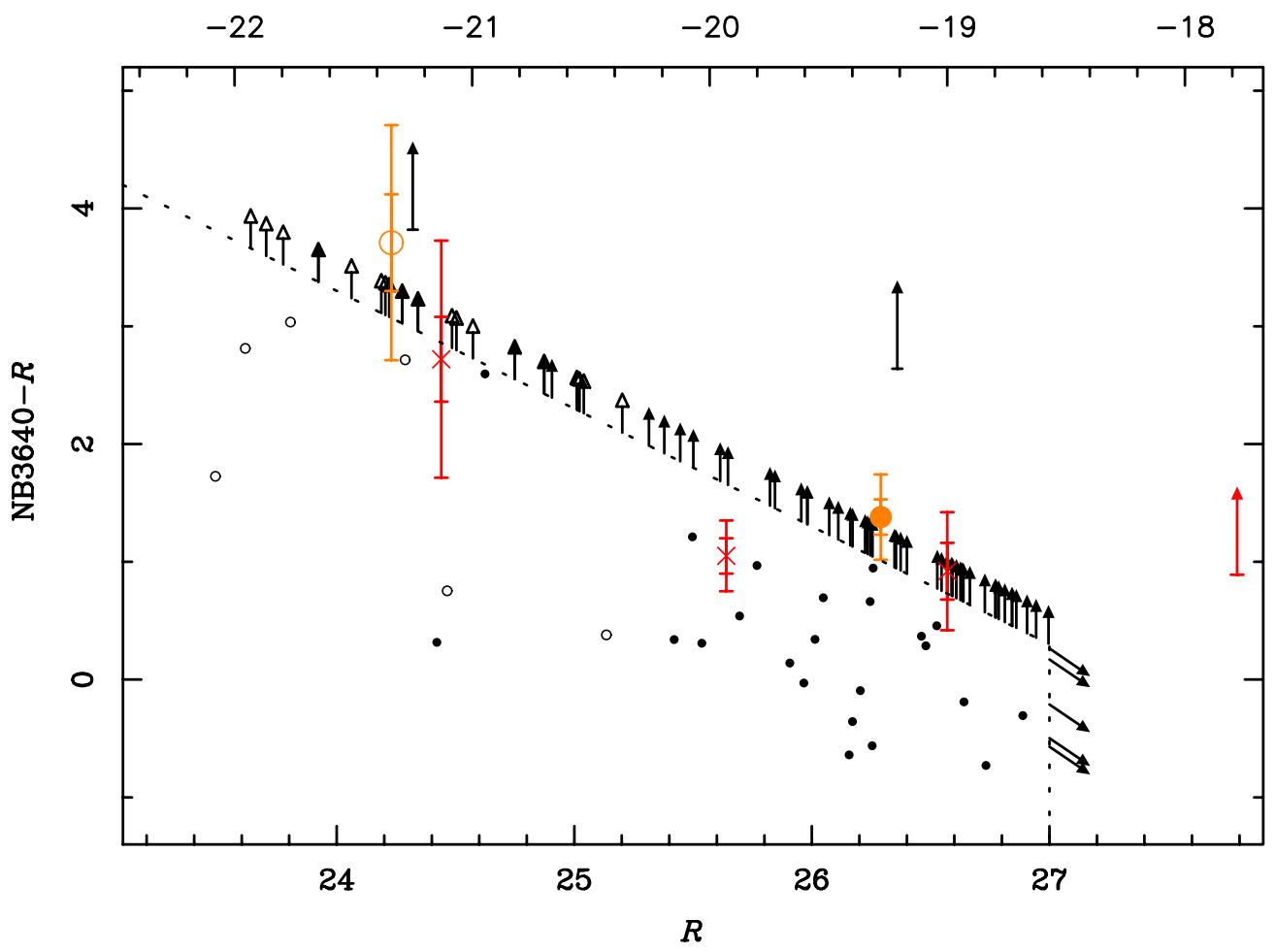

Figure 7. Rest-frame LyC-UV color vs. rest-frame UV magnitude. Open (filled) points and arrows represent LBGs (LAEs with $R$ and/or NB3640 detections). The uncertainty in color for fainter systems is fairly large; e.g., $\sigma_{\mathrm{NB} 3640-R} \gtrsim 0.5$ for $R \simeq 26.5$, NB3640-R $\simeq 0$ (see Table 2 ). Large points represent measurements made on stacks of the entire sample of LBGs and LAEs. Lower limits are for stacks of LBGs (far-left arrow) and LAEs (middle arrow) with no individual NB3640 detection. Smaller error bars indicate measurement uncertainty, while the larger bars also include sample variance computed through bootstrap resampling. Red crosses (and far-right lower limit) are measurements from stacks in bins of $R$ magnitude. Note that five of the eight LAEs in the brightest bin are also LBGs. The top axis is the corresponding absolute magnitude scale for $z=3.09$.

(A color version of this figure is available in the online journal.)

on their average escape fractions are $\left\langle f_{\mathrm{esc}}^{\mathrm{rel}}\right\rangle^{\text {non-det }}<0.5$ and $\left\langle f_{\text {esc }}^{\text {LyC }}\right\rangle^{\text {non-det }}<0.10$. Of the six LBGs with NB3640 detections, three have corrected UV-to-LyC flux density ratios consistent with $f_{\text {esc }}^{\text {rel }} \simeq 1$. However, the other three and the subsample average have NB3640- $R$ colors suggesting values greater than unity and therefore in conflict with current models. If we assume that LBGs with NB3640 detections preferentially sample clear sightlines through the IGM and we can thus ignore the IGM correction (but retain the statistical contamination correction) we find $\left\langle f_{\text {esc }}^{\text {rel }}\right\rangle^{\text {det }}>0.82(1 \sigma)$ and $\left\langle f_{\text {esc }}^{\text {LyC }}\right\rangle^{\text {det }}>0.18$ for the subsample of LBGs with NB3640 detections. For the LBG ensemble (detections and non-detections) average, the value of $\left\langle F_{\mathrm{UV}} / F_{\mathrm{LyC}}\right\rangle_{\text {corr }}^{\mathrm{LBG}}=11.3_{-5.4}^{+10.3}$ obtained by applying both IGM absorption and contamination corrections implies average escape fractions of $\left\langle f_{\text {esc }}^{\text {rel }}\right\rangle=0.53_{-0.25}^{+0.47}$ and $\left\langle f_{\text {esc }}^{\mathrm{LyC}}\right\rangle=0.12_{-0.06}^{+0.11}$. Note that all of the above uncertainties do not include contributions from the uncertainties in $\left(L_{\mathrm{UV}} / L_{\mathrm{LyC}}\right)_{\text {intr }}$ or $f_{\mathrm{esc}}^{\mathrm{UV}}$.

Due to the overall faintness of the LAE sample, we can place a meaningful lower limit on $f_{\text {esc }}^{\text {rel }}$ for only a few of the individual systems having no NB3640 detection. For the non-detection subsample average, we determine a weak upper limit of $\left\langle f_{\text {esc }}^{\text {rel }}\right\rangle \lesssim 1.2$ for our fiducial intrinsic Lyman break of six. The LAEs with detections have extremely blue NB3640- $R$ colors, however, implying individual $f_{\text {esc }}^{\text {rel }}$ values greater than unity at high significance for all but one system, even if we neglect corrections for IGM absorption. These extremely blue colors lead to an inferred ensemble relative escape fraction that is greater than unity at $\sim 4 \sigma$. However, an intrinsic value of $\left(L_{\mathrm{UV}} / L_{\mathrm{LyC}}\right)_{\text {intr }}=6$ may be high for the LAEs at $z \sim 3$, as their UV luminosity appears to be dominated by particularly young (Gawiser et al. 2007) and low-metallicity (Ono et al. 2010) populations. Thus, we also consider a lower intrinsic flux density ratio of three, which leads to $\left\langle f_{\mathrm{esc}}^{\mathrm{rel}}\right\rangle>0.75(2 \sigma)$, and $\left\langle f_{\text {esc }}^{\mathrm{LyC}}\right\rangle>0.17$ for the ensemble. We note, however, that as LAEs have been found to exhibit little reddening (Gawiser et al. 2007), $f_{\mathrm{esc}}^{\mathrm{UV}}$ is likely larger in our LAE sample than the fiducial LBG value of 20\%-25\%; thus, our determinations of $f_{\mathrm{esc}}^{\mathrm{LyC}}$ for LAEs should be considered conservative lower limits. Nonetheless, the NB3640- $R$ colors of LAEs with NB3640 detections are uncomfortably blue, even when neglecting the IGM-absorption corrections. The reasons for such small observed flux density ratios (see also Iwata et al. 2009; Inoue 2010; Inoue et al. 2011) in our LAE samples are not clear.

\subsection{The Space Density of Ionizing Photons at $z \simeq 3.09$}

Based on the average corrected UV-to-LyC flux density ratios for ensembles of LBGs and LAEs (Section 4.3), we can compute the comoving density of ionizing photons that escape from galaxies $z \simeq 3, \epsilon_{\mathrm{LyC}}=\left\langle F_{\mathrm{UV}} / F_{\mathrm{LyC}}\right\rangle_{\text {corr }}^{-1} \epsilon_{\mathrm{UV}}$, where $\epsilon_{\mathrm{UV}}$ is the comoving luminosity density in the non-ionizing UV continuum. The value of $\epsilon_{\mathrm{UV}}$ can be obtained by integrating the rest-frame UV LF. Thus,

$$
\epsilon_{\mathrm{LyC}}=\left\langle F_{\mathrm{UV}} / F_{\mathrm{LyC}}\right\rangle_{\mathrm{corr}}^{-1} \phi^{*} L^{*} \Gamma\left(2+\alpha, L_{\min } / L^{*}\right),
$$

where $\Gamma$ is the incomplete gamma function, $L^{*}$ is the luminosity corresponding to the characteristic magnitude $M^{*}$, and $L_{\min }$ is the minimum luminosity to which the LF is integrated. The $\lambda \sim 1700 \AA$ LF has been determined by Reddy et al. 


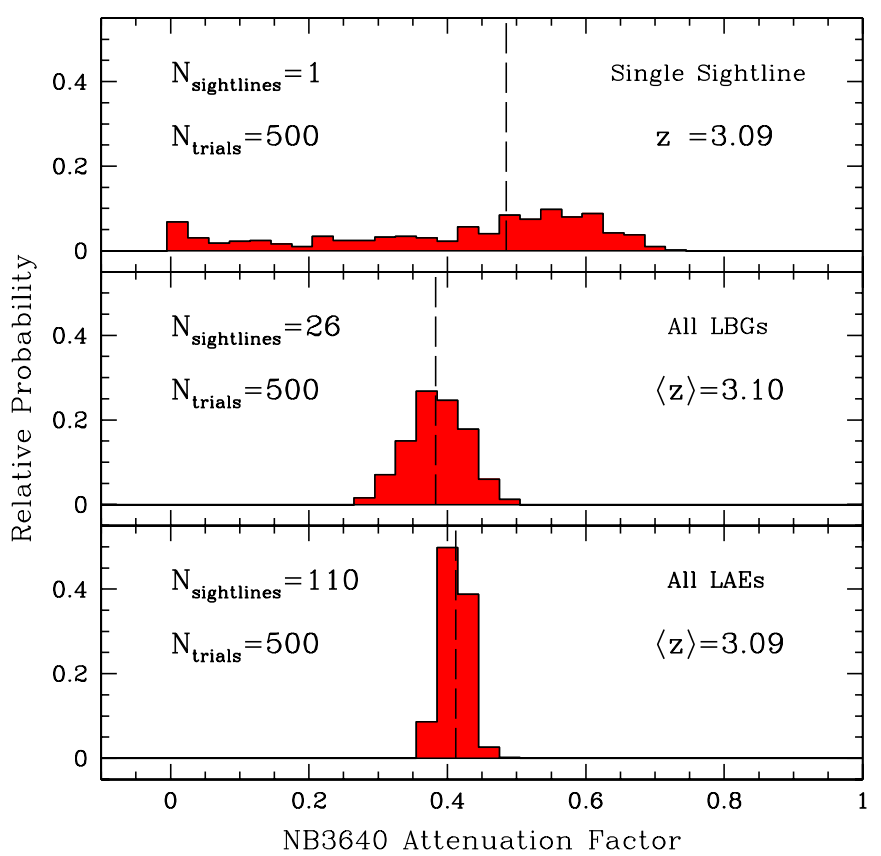

Figure 8. Results of Monte Carlo simulations of IGM opacity along the line of sight to our high-redshift targets. To account for IGM opacity, we generated a large number of random realizations of our measurements through the NB3640 filter. These simulations were tuned to the observation of an individual sightline (relevant for observations of individual LBGs and LAEs) as well as the average of ensembles of sightlines (relevant for sample averages and stacked images). In each simulation, using 500 random realizations, we recovered the distribution of the ratio between observed and unattenuated NB3640 flux, denoted here as "NB3640 Attenuation Factor." In each panel, the normalized distribution of simulated NB3640 attenuation factors is plotted, relevant for a given observed quantity. The dashed vertical line indicates the median NB3640 attenuation factor from each simulation, used to correct the observed individual or ensemble average $F_{\mathrm{UV}} / F_{\mathrm{LyC}}$ flux-density ratio for IGM attenuation. Top: the results for a single sightline to a source at $z=3.09$. Middle: the results for the ensemble average of $26 \mathrm{LBGs}$, with a mean redshift of $\langle z\rangle=3.10$. Bottom: the results for the ensemble average of $110 \mathrm{LAEs}$, with a mean redshift of $\langle z\rangle=3.09$.

(A color version of this figure is available in the online journal.)

(2008) for LBGs at $z \simeq 3$ with Schechter function parameters $\phi^{*}=1.66 \times 10^{-3} \mathrm{Mpc}^{-3}, M_{\mathrm{AB}}^{*}=-20.84, \alpha=-1.57$, and an effective redshift $z_{\text {eff }}=3.05$.

The average emergent UV-to-LyC flux density ratio for our sample of LBGs was determined from galaxies brighter than the photometric limit for spectroscopic confirmation of LBGs in the SSA22a field, $R=25.5$. This limit corresponds to $\simeq 0.5 L^{*}$ at $z \simeq 3$. Thus, we adopt $\left\langle F_{\mathrm{UV}} / F_{\mathrm{LyC}}\right\rangle_{\text {corr }}^{\mathrm{LBG}}=$ $11.3_{-5.4}^{+10.3}$ for galaxies with $\lambda \sim 1700 \AA$ continuum magnitudes $L \geqslant 0.5 L^{*}$. The galaxies in our LAE sample are generally fainter, reaching at least $R \simeq 27\left(\simeq 0.1 L^{*}\right)$. However, LAEs comprise only a subset of faint $z \sim 3$ galaxies and may provide a biased view of the average far-UV properties of galaxies with $\lambda \sim 1700 \AA$ continuum magnitudes of $0.1 L^{*} \leqslant$ $L<0.5 L^{*}$. We therefore estimate the contribution to $\epsilon_{\mathrm{LyC}}$ from fainter $\left(0.1 L^{*} \leqslant L<0.5 L^{*}\right)$ galaxies using in turn both the flux density ratio determined from our LBG sample $\left(\left\langle F_{\mathrm{UV}} / F_{\mathrm{LyC}}\right\rangle_{\text {corr }}^{\mathrm{LBG}}=11.3_{-5.4}^{+10.3}\right)$ and that determined from our LAE sample $\left(\left\langle F_{\mathrm{UV}} / F_{\mathrm{LyC}}\right\rangle_{\text {corr }}^{\mathrm{LAE}}=2.2_{-0.6}^{+0.9}\right)$. Using the LBGdetermined flux density ratio, we compute $\epsilon_{\mathrm{LyC}}\left(L \geqslant 0.5 L^{*}\right)=$ $7.7_{-3.7}^{+7.1} \times 10^{24} \mathrm{erg} \mathrm{s}^{-1} \mathrm{~Hz}^{-1} \mathrm{Mpc}^{-3}$ and $\epsilon_{\mathrm{LyC}}\left(0.1 L^{*} \leqslant L<\right.$ $\left.0.5 L^{*}\right)=9.2_{-7.2}^{+13.8} \times 10^{24} \mathrm{erg} \mathrm{s}^{-1} \mathrm{~Hz}^{-1} \mathrm{Mpc}^{-3}$, for a total of $\epsilon_{\mathrm{LyC}}\left(L \geqslant 0.1 L^{*}\right)=16.9_{-8.1}^{+15.5} \times 10^{24} \mathrm{erg} \mathrm{s}^{-1} \mathrm{~Hz}^{-1} \mathrm{Mpc}^{-3}$.

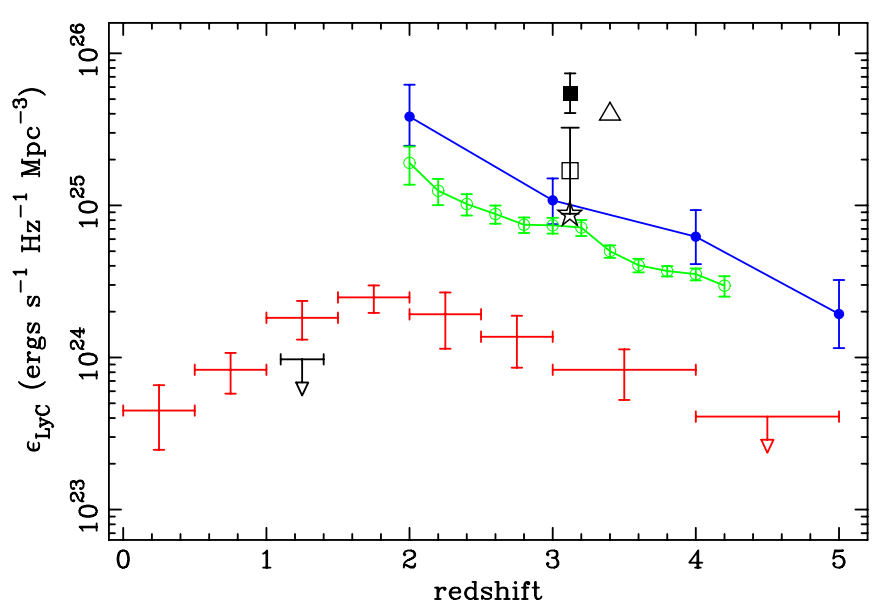

Figure 9. Contributions to the comoving emissivity of ionizing radiation at redshifts $0<z<5$. The open square is our determination of the contribution to $\epsilon_{\mathrm{LyC}}$ from galaxies with $L \geqslant 0.1 L^{*}$ assuming the estimate of the LBG flux density ratio holds over this luminosity range, while the filled square adopts our estimate of the LAE ratio for galaxies with $0.1 L^{*} \leqslant L<0.5 L^{*}$. Also shown are estimates based on the UV-to-LyC flux density ratios determined from: (star) spectra of 14 LBGs in the SSA22a field by Shapley et al. (2006); (triangle) the composite spectrum of 29 LBGs by Steidel et al. (2001); and (upper limit) rest-frame $\sim 700 \AA H S T$ images of 36 starburst galaxies at $z \approx 1.3$ by Siana et al. $(2007,2010)$. The red crosses are the contribution from broad-line QSOs taken from Cowie et al. (2009). The open and filled circles are estimates of the total ionizing emissivity determined using Equations (7) and (8) and estimates of the hydrogen photoionization rate in the Ly $\alpha$ forest, respectively, by Faucher-Giguère et al. (2008) and Bolton \& Haehnelt (2007). Upper limits are $3 \sigma$.

(A color version of this figure is available in the online journal.)

Considering instead the ratio determined from our LAE sample for the fainter galaxies, we find $\epsilon_{\mathrm{LyC}}\left(0.1 L^{*} \leqslant L<0.5 L^{*}\right)=$ $47.0_{-13.7}^{+17.6} \times 10^{24} \mathrm{erg} \mathrm{s}^{-1} \mathrm{~Hz}^{-1} \mathrm{Mpc}^{-3}$, for a total of $\epsilon_{\mathrm{LyC}}(L \geqslant$ $\left.0.1 L^{*}\right)=54.7_{-14.2}^{+19.0} \times 10^{24} \mathrm{erg} \mathrm{s}^{-1} \mathrm{~Hz}^{-1} \mathrm{Mpc}^{-3}$.

At $z \sim 3$, estimates of the UV-to-LyC flux density ratio in star-forming galaxies have been made by Steidel et al. (2001) using a composite spectrum of 29 LBGs and by Shapley et al. (2006) from spectra of 14 LBGs in the SSA22a field. We show our estimates of $\epsilon_{\mathrm{LyC}}\left(L \geqslant 0.1 L^{*}\right)$ in Figure 9, together with estimates that we derive using the flux density ratios given by Steidel et al. (2001) and Shapley et al. (2006) and assuming the same non-ionizing UV luminosity density from $L \geqslant 0.1 L^{*}$ galaxies that we computed above using the Reddy et al. (2008) LF. Additionally, using HST far-UV imaging of galaxies at $z \sim 1.3$, Siana et al. $(2007,2010)$ determined $(3 \sigma)$ lower limits of $\left\langle F_{\mathrm{UV}} / F_{\mathrm{LyC}}\right\rangle_{\text {corr }}>168$ and $\left\langle F_{\mathrm{UV}} / F_{\mathrm{LyC}}\right\rangle_{\text {corr }}>63$ on the average non-ionizing UV to $\sim 700 \AA$ flux density ratio for 15 $L \sim L^{*}$ starburst galaxies in the GOODS fields and 21 primarily $L<L^{*}$ galaxies in the HDF-N and HUDF, respectively. We also show the upper limit on $\epsilon_{\mathrm{LyC}}\left(L \geqslant 0.1 L^{*}\right)$ that we derive from their flux ratio limits in Figure 9, using the $\lambda \sim 1500 \AA \mathrm{LF}$ parameters determined at $z \sim 1$ from Arnouts et al. (2005) and integrating down to $L=0.1 L^{*}$. The contribution to $\epsilon_{\mathrm{LyC}}$ from broad-line QSOs determined by Cowie et al. (2009) is also shown, for comparison.

We can use our estimates of $\epsilon_{\mathrm{LyC}}$ to calculate the corresponding (proper) metagalactic specific intensity, $J_{v}$ :

$$
J_{v} \simeq(1+z)^{3} \epsilon_{\mathrm{LyC}} \frac{\lambda_{\mathrm{mfp}}}{4 \pi}
$$


Table 8

Contributions to the Ionizing Backgrounds

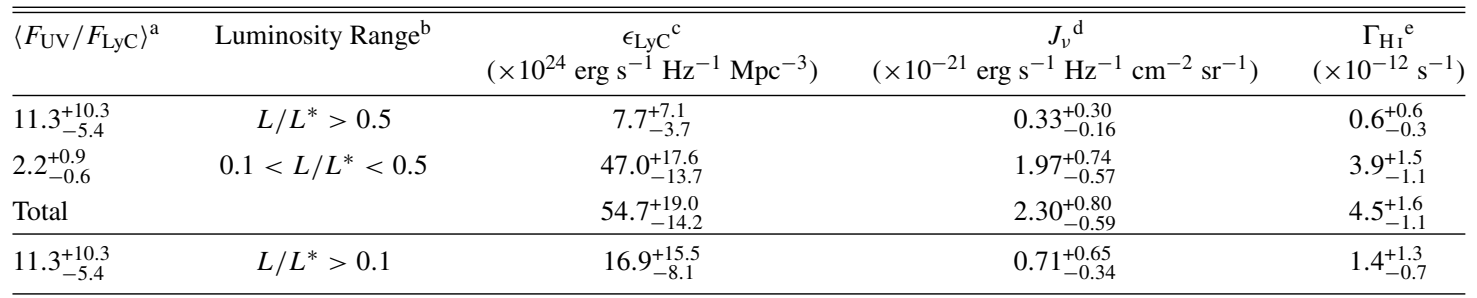

Notes.

${ }^{\text {a }}$ Corrected flux density ratio assumed for the luminosity range.

${ }^{b}$ Luminosity range over which the LF is integrated.

c Comoving specific emissivity of ionizing radiation.

${ }^{\mathrm{d}}$ Proper metagalactic specific intensity of ionizing radiation.

${ }^{\mathrm{e}}$ Inferred contribution to the intergalactic hydrogen photoionization rate from galaxies in the luminosity range.

We find $J_{v}\left(L \geqslant 0.5 L^{*}\right)=0.33_{-0.16}^{+0.30} \times 10^{-21} \mathrm{erg} \mathrm{s}^{-1} \mathrm{~Hz}^{-1}$ $\mathrm{cm}^{-2} \mathrm{sr}^{-1}$ and, assuming the LBG flux density ratio holds down to $L=0.1 L^{*}, J_{v}\left(L \geqslant 0.1 L^{*}\right)=0.71_{-0.34}^{+0.65} \times 10^{-21} \mathrm{erg} \mathrm{s}^{-1}$ $\mathrm{Hz}^{-1} \mathrm{~cm}^{-2} \mathrm{sr}^{-1}$. Alternatively, computing the contribution from fainter galaxies assuming the flux density ratio determined from our LAE sample leads to $J_{v}\left(L \geqslant 0.1 L^{*}\right)=2.30_{-0.59}^{+0.80} \times 10^{-21}$ erg s${ }^{-1} \mathrm{~Hz}^{-1} \mathrm{~cm}^{-2} \mathrm{sr}^{-1}$.

Furthermore, if we assume a form for the spectrum of escaping ionizing flux, we can estimate the contribution to the hydrogen photoionization rate $\Gamma_{\mathrm{H}}$ in the IGM from star-forming galaxies at $z \simeq 3$. The photoionization rate is of particular interest as $\Gamma_{\mathrm{H}_{\mathrm{I}}}$ has been inferred in an independent manner from Ly $\alpha$ forest data (e.g., Bolton et al. 2005; Faucher-Giguère et al. 2008). If we assume a power-law spectrum $J_{v}=J_{v_{0}}\left(v / \nu_{0}\right)^{\alpha_{s}}$, where $\nu_{0}$ corresponds to the Lyman limit and $\sigma_{\mathrm{HI}}=6.3 \times 10^{-18}$ $\mathrm{cm}^{-2}$ is the atomic hydrogen photoionization cross section at $v_{0}$, then

$$
\Gamma_{\mathrm{HI}}=\frac{4 \pi \sigma_{\mathrm{HI}} J_{v}}{h\left(3-\alpha_{s}\right)}
$$

where $h$ is Planck's constant. The intrinsic spectrum of starforming galaxies below the Lyman limit is poorly constrained. Typical values adopted in past work have ranged from $\alpha_{s}=-3$ (Bolton \& Haehnelt 2007; Ouchi et al. 2009) to $\alpha_{s}=+0.5$ (Faucher-Giguère et al. 2008). If we assume a value of $\alpha_{s}=-3$ we find a contribution of $\Gamma_{\mathrm{HI}}=0.6_{-0.3}^{+0.6} \times 10^{-12} \mathrm{~s}^{-1}$ from $L \geqslant 0.5 L^{*}$ galaxies. For the full range of $L \geqslant 0.1 L^{*}$, we determine $\Gamma_{\mathrm{HI}}=1.4_{-0.7}^{+1.3} \times 10^{-12} \mathrm{~s}^{-1}$ using the LBG flux density ratio, or, computing the contribution for faint galaxies using the LAE ratio, $\Gamma_{\mathrm{HI}_{\mathrm{I}}}=4.5_{-1.1}^{+1.6} \times 10^{-12} \mathrm{~s}^{-1}$. Estimates of $\Gamma_{\mathrm{HI}}$ from Ly $\alpha$ forest optical depths range from $\simeq 0.6 \times 10^{-12} \mathrm{~s}^{-1}$ (FaucherGiguère et al. 2008) to $\simeq 1.3 \times 10^{-12} \mathrm{~s}^{-1}$ (Kirkman et al. 2005). The value obtained by applying the LBG flux density ratio over $L>0.1 L^{*}$ is consistent with this range, while attributing the LAE flux density ratio to fainter galaxies results in an estimate that is $3 \sigma$ larger than implied by the Ly $\alpha$ forest data. In Figure 9, we show the values of $\epsilon_{\mathrm{LyC}}$ corresponding to the determinations of $\Gamma_{\mathrm{HI}}$ by Bolton \& Haehnelt (2007) and Faucher-Giguère et al. (2008) from $z=2$ to $z \sim 5$. We arrive at these values using Equations (7) and (8) together with our adopted values of $\alpha_{S}$ and $\lambda_{\mathrm{mfp}}$. Our estimates of $\epsilon_{\mathrm{LyC}}, J_{v}$, and $\Gamma_{\mathrm{H}_{\mathrm{I}}}$ are summarized in Table 8.

Finally, it should be noted that the values estimated in this subsection are sensitive to the adopted parameters. Specifically, integrating the LF down to $L_{\min }=0$ would result in a factor of two increase in $\epsilon_{\mathrm{LyC}}, J_{v}$, and $\Gamma_{\mathrm{HI}}$ compared to an integration lower limit of $L_{\text {min }}=0.1 L^{*}$. Using the UV LF of Reddy \& Steidel (2009) would not change our estimates of the contribution to $\epsilon_{\mathrm{LyC}}, J_{v}$, and $\Gamma_{\mathrm{H}_{\mathrm{I}}}$ from galaxies with $L \geqslant 0.5 L^{*}$, but would increase our estimates for the ranges $L \geqslant 0.1$ and $0.1 L^{*} \leqslant L<0.5 L^{*}$ by $14 \%$ and $27 \%$, respectively. Contrastingly, using the $L y A$ offsets to determine the contamination correction for the LAEs (Section 3.5) would decrease our estimates of the LAE contribution to $\epsilon_{\mathrm{LyC}}, J_{v}$, and $\Gamma_{\mathrm{HI}}$ by $\simeq 15 \%$, while adopting a far-UV spectral slope of $\alpha_{s}=+0.5$ would increase our estimates of $\Gamma_{\mathrm{HI}}$ by a factor of 2.4.

\section{DISCUSSION}

\subsection{Summary of the $L B G / L A E$ Subsamples}

\subsubsection{LBGs}

Of the 26 LBGs at $z \geqslant 3.06$ in the field of our NB3640 image, we have detected 6 down to a limiting magnitude of 27.3. Correcting for foreground contamination and absorption by the neutral IGM, we determine $\left\langle F_{\mathrm{UV}} / F_{\mathrm{LyC}}\right\rangle_{\text {corr }}^{\mathrm{LBG}}=11.3_{-5.4}^{+10.3}$. Previous direct measurements of the relative UV-to-LyC emergent flux density ratios at $z \sim 3$ have found values ranging from $\simeq 4$ (Steidel et al. 2001; Iwata et al. 2009) to $\simeq 20$ (Shapley et al. 2006). Our value is roughly consistent with both extremes. For an intrinsic ratio of six and a non-ionizing UV escape fraction of $\sim 20 \%-25 \%$, this range in $\left\langle F_{\mathrm{UV}} / F_{\mathrm{LyC}}\right\rangle_{\text {corr }}$ corresponds to a range of $0.07-0.4 \mathrm{in}\left\langle f_{\mathrm{esc}}^{\mathrm{LyC}}\right\rangle$, with our value giving $\left\langle f_{\mathrm{esc}}^{\mathrm{LyC}}\right\rangle=0.12_{-0.06}^{+0.11}$.

Monte Carlo simulations based on the distribution of offsets between the NB3640 and $R$-band detections indicate that two to four of the LBGs with NB3640 detections are uncontaminated by foreground sources. Thus, our corrected LBG detection rate is $8 \%-15 \%(1 \sigma)$. Three of the detections have ratios of nonionizing to ionizing flux densities that are in tension with that allowed by stellar population models. If we take the most conservative interpretation of our results and consider only C49 and D17-which have $\Delta_{R}<1^{\prime \prime}$ and $f_{\text {esc }}^{\text {rel }} \lesssim 1$ - as uncontaminated detections, we arrive at an ensemble $\left\langle f_{\mathrm{esc}}^{\text {rel }}\right\rangle^{\mathrm{LBG}} \simeq 0.10$ and $\left\langle f_{\mathrm{esc}}^{\mathrm{LyC}}\right\rangle^{\mathrm{LBG}} \simeq 0.02$. However, our contamination simulations suggest that this scenario is overly conservative.

Other authors have made indirect measurements of the $\mathrm{LyC}$ escape fraction at $z \sim 3$. Faucher-Giguère et al. (2008) predict $\left\langle f_{\mathrm{esc}}^{\mathrm{LyC}}\right\rangle \sim 5 \times 10^{-3}$ to account for the ionizing background inferred from the photoionization rate in the Ly $\alpha$ forest. However, 


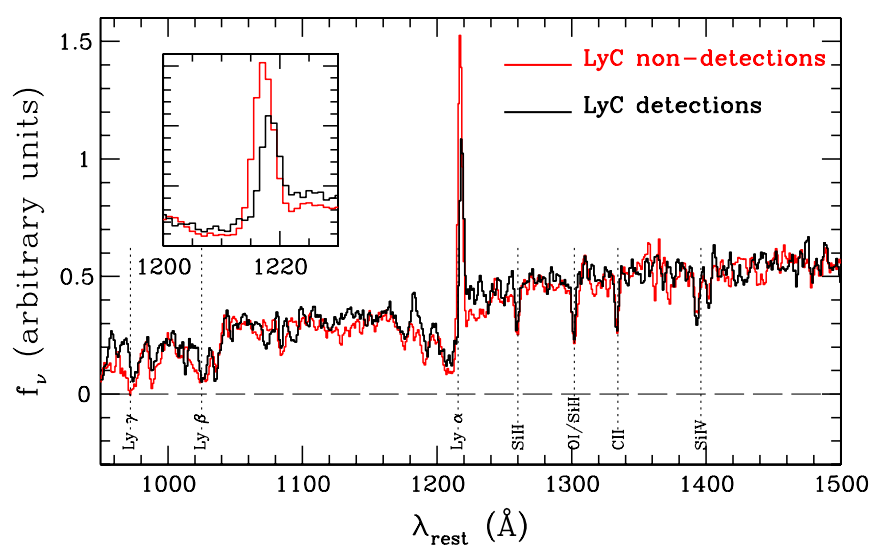

Figure 10. Comparison of composite rest-frame UV spectra for the 6 LBGs with LyC detections in the NB3640 filter (black), and the 20 objects without detections (red). The composite spectra have been scaled to a common value over 1400-1500 A. In terms of overall spectral shape and strength of interstellar absorption features, the two spectra are very similar. The only notable difference is observed in the strength of Ly $\alpha$ emission (indicated in greater detail in the inset panel). The spectrum of NB3640 non-detections has a Ly $\alpha$ equivalent width $\sim 2-3$ times larger than that of the detections. At the same time, the centroid of the Ly $\alpha$ profile in the spectrum of the detections is shifted toward longer wavelengths.

(A color version of this figure is available in the online journal.)

their estimate is averaged over all galaxies down to $L_{\mathrm{UV}}=0$, assumes an intrinsic UV-to-LyC flux density ratio of unity, and is computed using an ionizing spectral slope of $\alpha_{s}=+0.5$; all of these assumptions decrease the escape fraction needed to match the photoionization rate. Chen et al. (2007) use spectra of long-duration $\gamma$-ray bursts to determine the column densities of neutral hydrogen along the sightline though the ISM. Their results suggest $\left\langle f_{\mathrm{esc}}^{\mathrm{LyC}}\right\rangle=0.02$ in the sub- $L^{*}$ galaxies that are expected to dominate their sample. It is unclear if the difference in the various estimates of the escape fraction is due to subtleties in the methods or differences in the samples. For example, it could be that $\left\langle f_{\mathrm{esc}}^{\mathrm{LyC}}\right\rangle$ depends on environment or galaxy type.

Our sample of NB3640-detected LBGs is small, and likely $\sim 50 \%$ contaminated by non-detections. Thus, any differences in the average rest-frame non-ionizing UV properties between galaxies with and without leaking LyC flux may not be evident in our data unless the trends are particularly strong. Nonetheless, we investigated the UV properties of the LBGs in our sample by creating stacks of LRIS spectra (Shapley et al. 2003, 2006) for galaxies with and without NB3640 detections, which we show in Figure 10. ${ }^{7}$ We find no significant differences between the two samples in either their average spectral slopes or interstellar absorption lines, although the strength of the Ly $\alpha$ emission line is on average weaker in our LBGs with NB3640 detections. The LBGs undetected in NB3640 are also slightly fainter on average, consistent with our findings from the stacked $R$-band photometry (Table 6). We also estimate the average UV slopes of the two subsamples photometrically, both through aperture photometry on stacked images in the $V$ and $R$ bands, and through averages of the individual fluxes after correcting the $V$ band magnitudes for Ly $\alpha$ emission and IGM absorption. With

\footnotetext{
7 We note that the offset of the NB3640 detection from the $R$-band position in some LBGs is significant compared to the 1 ". 2 slits through which the LRIS spectra were obtained. Thus, the stellar populations associated with the NB3640 emitting regions may not contribute to the observed spectra in these cases.
}

neither method do we find significant differences in the average $V-R$ colors between the two subsamples. We do note that the 26 LBGs in our sample are on average slightly bluer in $G-R$ (corrected for Ly $\alpha$ emission and IGM absorption) than LBGs in the large spectroscopic sample presented in Shapley et al. (2003), which highlights the need for a larger "average" sample.

\subsubsection{LAES}

We detected 27 of the 110 main sample LAEs in the NB3640 image. Our Monte Carlo simulations suggest that 18-24 of the detections are uncontaminated, leading to a corrected LAE detection rate of $16 \%-22 \%$. After applying contaminationand IGM absorption-corrections we determine an ensemble $\left\langle F_{\mathrm{UV}} / F_{\mathrm{LyC}}\right\rangle_{\text {corr }}^{\mathrm{LAE}}=2.2_{-0.6}^{+0.9}$. Most of our LAEs with individual detections have flux density ratios inconsistent with predictions of stellar population models even for young, low-metallicity populations and neglecting the IGM absorption correction. In contrast, the lower limit on the average UV-to-LyC flux density ratio for LAEs without individual NB3640 detections $\left(\left\langle F_{\mathrm{UV}} / F_{\mathrm{LyC}}\right\rangle_{\mathrm{obs}}^{\mathrm{LAE}, \text { non-det }}>12.2\right)$ is more than a factor of nine higher than the average observed ratio for those with detections $\left(\left\langle F_{\mathrm{UV}} / F_{\mathrm{LyC}}\right\rangle_{\text {obs }}^{\mathrm{LAE}, \mathrm{det}}=1.3\right)$, implying a dichotomy in the far-UV properties of $z \simeq 3$ LAEs.

The implied relative escape fractions $f_{\text {esc }}^{\text {rel }} \gtrsim 1$ for our LAEs detected in NB3640 are difficult to explain. Exotic stellar population models with top-heavy IMFs and extremely low metallicities could in principle reproduce the observed flux density ratios (e.g., Inoue et al. 2011), though we caution that there is little additional evidence to suggest such models are appropriate for $z \sim 3$ LAEs. As with the LBGs, we find no significant differences between the average nonionizing UV slopes of LAEs with and without NB3640 detections, determined either via aperture photometry on the $R$ - and $V$-band stacks, or via Ly $\alpha$-corrected averages of the individual $V$ and $R$ magnitudes. In Figure 11, we show the Ly $\alpha$ rest-frame equivalent widths for our LAE sample, estimated from their $B V-\mathrm{NB} 3980$ colors, as a function of UV continuum (i.e., $R$-band) magnitude. The largest equivalent widths tend to be confined to fainter continuum sources, consistent with past findings (e.g., Ando et al. 2006; Stark et al. 2010). In order to be detected in NB3640, the faintest sources require lower non-ionizing UV-to-LyC flux density ratios (e.g., see Figure 7). Thus, any comparison of the distributions of equivalent widths between sources with and without NB3640 detections should be confined to a relatively narrow range of continuum magnitude. The inset in Figure 11 shows such distributions for the ranges $R<26.1$ and $26.1<R<27$, demonstrating that the median $\mathrm{Ly} \alpha$ equivalent widths are smaller for LAEs detected in NB3640 compared to those without detections.

\subsubsection{Faint LAEs}

In addition to our main sample LAEs, we have identified 20 "faint" LAE candidates with $26<\mathrm{NB} 4980 \leqslant 26.5$ and $B V-\mathrm{NB} 4980 \geqslant 1.2$. These faint LAEs have larger photometric uncertainties (e.g., see Table 2). As they have different selection criteria from the main sample and are detected at lower significance, we have not included them in our statistical analyses. However, for completeness we present results for the average properties of this sample from our stacking analysis in Table 6 and for individual objects in Table 10. 


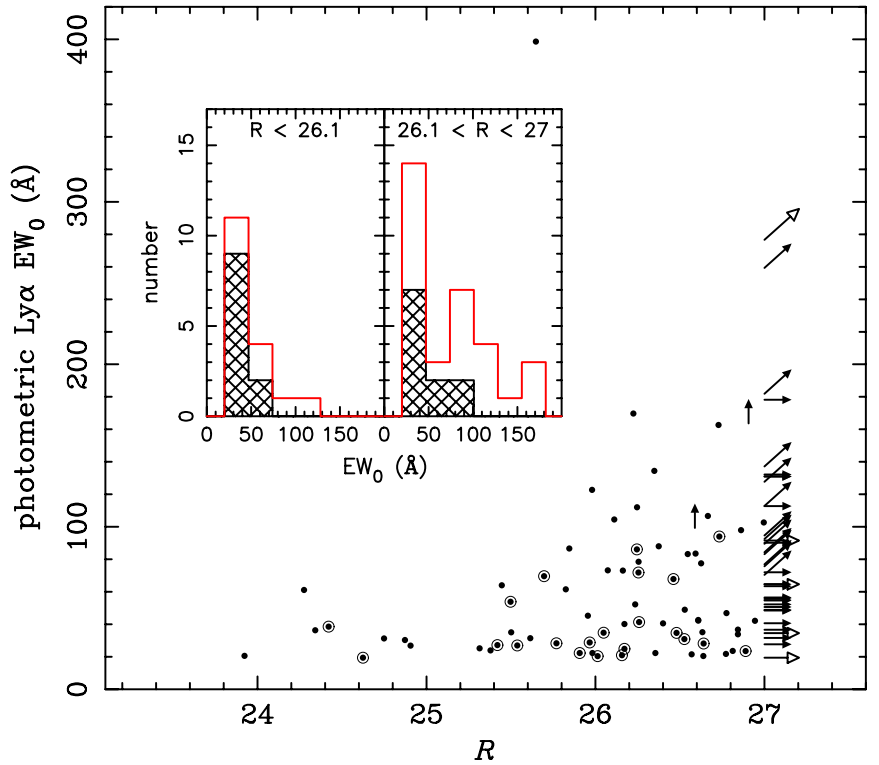

Figure 11. LAE Ly $\alpha$ rest-frame equivalent widths determined from $B V-\mathrm{NB} 4980$ colors. Sources detected in NB3640 are indicated by circled points and open arrows (plotted slightly larger than solid arrows for clarity). Consistent with previous studies, we find that the highest equivalent widths tend to be associated with sources that are fainter in the rest-frame UV continuum. The inset shows the distribution of equivalent widths for brighter (left) and fainter (right) LAEs; the median $R$-band magnitude for sources with $R$ band detections is $R=26.1$. Sources with and without NB3640 detections are represented by the black hashed and red histograms, respectively.

(A color version of this figure is available in the online journal.)

We detect only one object in the faint sample in NB3640, while in the main sample we detect $14 \%$ of the 65 LAEs having $B V-\mathrm{NB} 4980 \geqslant 1.2$ in the NB3640 image. However, the faint LAEs are also on average fainter in $R$ and therefore have smaller detection limits in $F_{\mathrm{UV}} / F_{\mathrm{LyC}}$. Thus, the data do not allow us to address any statistical differences between the two populations.

\subsection{Other Considerations}

\subsubsection{Are We Missing Low $\left(F_{\mathrm{UV}} / F_{\mathrm{LyC}}\right)_{\mathrm{obs}} L B G s$ ?}

Of the 110 LAEs in our sample, $24 \%$ have NB3640-R $<1.5$, compared to $8 \%$ of our LBGs. The Lyman break technique specifically selects against galaxies with notably blue broadband $U_{n}-R$ colors (Steidel et al. 2003), and while it is possible for a $z \geqslant 3.06$ galaxy to have significantly higher flux density through the narrow NB3640 filter than through the relatively broad $U_{n}$ band, we note that the effective wavelengths of the two filters are very similar. The LAEs discussed here, on the other hand, are selected by their $\operatorname{Ly} \alpha$ emission line strength independently of their rest-frame UV colors and corresponding $\left(F_{\mathrm{UV}} / F_{\mathrm{LyC}}\right)$ values. Therefore, we must consider whether the very application of the Lyman break technique selects against a significant number of non-LAE galaxies with $R$ magnitudes similar to our LBG sample but NB3640- $R$ colors similar to those observed in our LAE sample. If such a population exists at $z \sim 3$, it would appear as a high-redshift tail in samples of BX/BM galaxies (Steidel et al. 2004; Reddy et al. 2008) selected to target galaxies with bluer $U_{n}-R$ colors at $1.4 \lesssim z \lesssim 2.5$. However, the fraction and associated surface density of such galaxies with $z \geqslant 3.06$ is exceedingly small (see, e.g., Figure 1 of Reddy et al. 2008), and the incidence of such systems is likely consistent with foreground contamination. Thus, while the nature of the high- $z$ BX/BM galaxies should be explored further, it does not appear that we are missing significant numbers of rest-frame UV bright galaxies having NB3640- $R \sim 0$ due to our selection of $z \sim 3$ galaxies using the Lyman break technique.

\subsubsection{Viewing Angle Effects}

As with the bulk of our $z \simeq 3$ sample, searches for emerging LyC photons from galaxies at lower redshifts have largely yielded null results (Malkan et al. 2003; Siana et al. 2007, 2010; Bridge et al. 2010). The small fraction of star-forming galaxies with detected LyC emission implies that, for ionizing radiation to escape, there must be pathways through the neutral ISM from star-forming regions that are unusually clear of dust and gas. Processes capable of removing or destroying neutral gas and dust (e.g., feedback, tidal stripping, etc.) in actively star-forming galaxies are unlikely to be sufficiently effective to allow the transmission of ionizing radiation over all solid angle. Large spatial variations in $f_{\mathrm{esc}}^{\mathrm{LyC}}$ are also predicted by simulations (e.g., Razoumov \& Sommer-Larsen 2007; Gnedin et al. 2008). If LyC flux only escapes over a small fraction of the total solid angle relative to the non-ionizing flux, this would naturally explain the large spread in the observed far-UV properties in our samples.

We consider a simple model in which most of the UV and LyC flux emitted in directions of cleared ISM escapes while some UV and no LyC flux escapes in other directions. If the projected area of the cleared ISM is comparable to the size of the LyC-emitting region, the relative escape fraction from that region would either be negligible or near unity, depending on whether the viewing angle samples a direction through the cleared ISM. An ensemble of galaxies having a single, compact starburst would then be expected to exhibit a bimodal observed distribution of relative escape fractions. In contrast, a single projected view of a galaxy having multiple or extended regions of star formation could sample regions both cleared of and obscured by neutral ISM, effectively averaging out the bimodality inherent in compact systems. A wider range of observed values for the relative escape fraction would then be possible for such a galaxy. Based on the ACS-F814W images in Figures 3 and 4(a)-(e), the LAEs in our sample appear to be on average more compact than the LBGs and, consistent with this simple picture, more bimodal in their LyC properties relative to the LBGs.

If the escape of LyC photons is highly anisotropic, then the observed UV-to-LyC flux density ratio in an individual source is unlikely to be representative of the ratio of the integrated (i.e., over all solid angle) UV-to-LyC flux densities escaping from that source. For a sample large enough to contain a significant number of LyC detections, however, the ensemble average ratios should reflect the average integrated flux density ratios. Additionally, under the assumption that all galaxies in each subsample have similar properties, the detection rate should indicate the average fraction of solid angle over which LyC photons can escape a typical star-forming galaxy at $z \simeq 3$. Of course, if there are evolutionary effects within our LBG and LAE samples, the assumption of equivalence within each galaxy sample is not valid. Careful consideration of the stellar populations of the sources is required to test this assumption (C. C. Steidel et al. 2011, in preparation). For now we note that large differences are not apparent in the average 
UV properties of LBGs or LAEs with and without NB3640 detections.

\subsubsection{IGM Coherence Effects}

When determining the uncertainty in the IGM absorption corrections for the ensemble samples, we assume the IGM properties along the sightlines to each source are uncorrelated. If this is not an adequate assumption, the uncertainties in our computed escape fractions and IGM-corrected emissivities may be underestimated. Indeed, studies have found coherence in the Ly $\alpha$ forest over Mpc scales (Becker et al. 2004; Casey et al. 2008) at $z \sim 3$, though correlations are weaker in the higher column density systems relevant to our corrections and a detailed description of the sizes and correlation scales of forest clouds is still lacking (see, e.g., Meiksin 2009). Furthermore, there may be systematic environmental effects that we are not considering. For example, the fact that our targets lie in or behind a largescale galaxy overdensity (Steidel et al. 2000) may cause the IGM to be on average more opaque than expected with a higher than average incidence of LLSs. Alternatively, a proximity effect due to the relatively high density of star-forming galaxies and QSOs in the protocluster could in principle diminish the IGM opacity on Mpc scales making the sightlines to our targets less opaque to ionizing radiation. It should be noted, however, that the UV-to-LyC flux density ratios in our LAEs with detections are uncomfortably small even before the IGM corrections are made.

\subsection{Implications}

Our results suggest the following picture. Neutral gas and dust in the ISM of typical star-forming galaxies at $z \sim 3$, as traced by LBGs, reprocesses most of the non-ionizing UV flux, and effectively all of the LyC flux, over most solid angle. Over some significant fraction of solid angle $(\sim 10 \%-20 \%$, as suggested by our corrected detection rates), however, the neutral gas and dust have been cleared or destroyed, allowing both ionizing and non-ionizing flux to escape into the IGM in those directions. Such regions are likely to be smaller than the projected UV sizes of typical bright galaxies but may be comparable to the sizes of LyC-emitting regions in compact sources. In this picture, when detected in $\mathrm{LyC}$, compact sources should on average exhibit larger relative escape fractions compared to LyC-detected galaxies with extended starforming regions. Sightlines to relatively faint, low metallicity star-forming sources, such as may be selected by our LAE sample, could have lower intrinsic UV-to-LyC ratios further boosting the observed non-ionizing to ionizing UV flux density ratios.

We are left with two difficulties in this picture, however. First, the observed LyC flux density relative to the non-ionizing UV continuum for the majority of our sources with putative LyC detections exceeds predicted unattenuated ratios for current reasonable stellar population models. Second, even if such ratios were possible to explain, the implied contribution to the intergalactic neutral hydrogen photoionization rate from LAEs is in excess of that determined from measurements of the opacity in the Ly $\alpha$ forest (Bolton et al. 2005; FaucherGiguère et al. 2008). A satisfactory model that can reproduce all of the observational evidence is still lacking and followup spectroscopy (e.g., Inoue et al. 2011) is needed to determine the nature of the NB3640 detections associated with our LAEs.
The LyC photons that escape from galaxies prior to the end of reionization cannot be directly observed, as they are absorbed during the reionization process (Fan et al. 2006; Robertson et al. 2010). Even after the epoch of reionization, at redshifts $z \gtrsim 4$, the opacity of the IGM to ionizing photons due to overdensities retaining significant neutral fractions is sufficient to make direct detection of escaping LyC flux improbable (Inoue \& Iwata 2008; Vanzella et al. 2010b). Thus, determinations of $f_{\mathrm{esc}}^{\mathrm{LyC}}$ from direct measurements at $z \approx 3$ are likely to be the best constraint on the LyC escape fraction for the galaxies that presumably reionized the universe. Gnedin (2008) determined that, if the LyC escape fraction for low mass $\left(M_{\text {halo }}<10^{11} M_{\odot}\right)$ galaxies is negligible, there would be insufficient LyC photons for reionization. On the contrary, our results for LAEs (while puzzling) imply that relatively faint galaxies on average have $\mathrm{LyC}$ escape fractions larger than those of bright galaxies. Recent data obtained with the Wide Field Camera 3 on HST have allowed for the study of galaxies out to redshifts $z \sim 7$ (e.g., Bunker et al. 2010; Oesch et al. 2010). Bouwens et al. (2010) find a steep faint-end slope to the $z \sim 7 \mathrm{LF}, \alpha=-1.94$. Parameterizing the observed evolution of the LF from $z \sim 4$ to $z \sim 8$, they extrapolate the LF to even higher redshift and claim that the universe can be reionized by $z \sim 6$ if the average escape fraction is $\left\langle f_{\text {esc }}^{\mathrm{LyC}}\right\rangle=0.2$. This result is in agreement with earlier findings by Ouchi et al. (2009). If $\left\langle f_{\mathrm{esc}}^{\mathrm{LyC}}\right\rangle=0.6$, Bouwens et al. (2010) claim that reionization is complete by $z \sim 8$ and the predicted Thomson optical depth would be within $1 \sigma$ of that measured for the $W M A P$ seven-year data set. Since the luminosity density at these redshifts is apparently dominated by relatively faint galaxies, the LyC escape fraction in fainter sources such as our sample of LAEs is of particular importance. Resolving the discrepancy between the theoretical minimum and observed UVto-LyC flux density ratios is thus an important undertaking for future work.

\section{SUMMARY}

We have presented analysis of the deepest near-UV image to date of the SSA22a field. This image was obtained through a narrowband filter sampling the redshifted LyC emission from $z \geqslant 3.06$ sources, including many galaxies in the $z=$ $3.09 \pm 0.03$ SSA22a protocluster. The image contains 26 LBGs with spectroscopically determined redshifts $z \geqslant 3.06$ for which our NB3640 filter is uncontaminated by emission longward of the redshifted Lyman limit. We augmented these data with both new and archival deep narrowband and broadband images designed to select LAEs at $3.06 \lesssim z \lesssim 3.12$. From these images we have identified 110 LAE candidates. Our main conclusions are as follows.

1. Six of the 26 LBGs have NB3640 detections with $A B$ magnitudes in the range 25.2 to 27.0 . Five of the detections are offset from the $R$-band detections by $\leqslant 1^{\prime \prime}$. We have used Monte Carlo simulations to determine the probability of contamination by foreground objects and conclude that two to four ( $1 \sigma$ confidence interval) of the six detections are uncontaminated. Twenty-seven of the 110 LAEs are detected in NB3640 with magnitudes spanning 24.7 to 27.2, twenty of which are offset from the $R$-band detection by $\leqslant 1^{\prime \prime}$. Our simulations suggest that $18-24$ of our LAEs with NB3640 detections are uncontaminated.

2. For our sample of LBGs we find a large range in NB3640- $R$ colors and thus observed UV-to-LyC flux density ratios. Furthermore, the subsample of LAEs having no individual 
NB3640 detection has a lower limit on the average UV-toLyC flux density ratio that is almost an order of magnitude larger than observed for LAEs with NB3640 detections, implying a significant dichotomy in the observed far-UV properties of LAEs. We interpret this variety in the observed LyC properties of $z \approx 3$ galaxies as arising from the partial clearing of neutral gas and dust over a limited solid angle in individual systems. In this picture, ensemble measurements of $f_{\mathrm{esc}}^{\mathrm{LyC}}$ (when corrected for foreground contaminants and IGM absorption) will produce accurate average values, while measurements in individual systems will be highly biased by orientation.

3. We find no difference between the average non-ionizing rest-frame UV properties for LBGs with and without NB3640 detections, with the possible exception that the Ly $\alpha$ emission line may be weaker in those with detections. We also find no difference in the average UV spectral slopes for LAEs with and without NB3640 detections, although we again find evidence for weaker $\operatorname{Ly} \alpha$ emission in LAEs with detections. The similar UV properties of the two samples supports the idea that orientation effects determine the detectability of escaping LyC flux, if such affects are less important for the non-ionizing UV compared to the LyC.

4. After applying statistical corrections for foreground contaminations and IGM absorption, and assuming an intrinsic UV-to-LyC flux density ratio of six and a UV escape fraction of $\sim 20 \%-25 \%$, we determine $f_{\mathrm{esc}}^{\mathrm{LyC}} \sim 0.1$ for our sample of LBGs. The LBGs in our sample represent the bright end $\left(L \gtrsim 0.5 L^{*}\right)$ of the rest-frame UV LF at $z \approx 3$. If their average UV-to-LyC flux density ratio is representative of LBGs down to $0.1 L^{*}$, their inferred contribution to the intergalactic hydrogen photoionization, $\Gamma_{\mathrm{HI}}$, rate is consistent (within the large uncertainties) with that measured from the Ly $\alpha$ forest opacity (Bolton et al. 2005; Kirkman et al. 2005; Faucher-Giguère et al. 2008).

5. Half of our LBGs and all but one of our LAEs with NB3640 detections have IGM-corrected UV-to-LyC flux density ratios significantly smaller than the minimum predicted from stellar population models. Our LBGs are drawn from the bright end of the LBG LF, $L \geqslant 0.5 L^{*}$. If the average LAE UV-to-LyC flux density ratio that we measure is representative of LBGs over the magnitude range of our LAEs, $0.1 L^{*} \lesssim L \lesssim 0.5 L^{*}$, then the inferred contribution to the global hydrogen photoionization rate $\Gamma_{\mathrm{H}_{\mathrm{I}}}$ exceeds the values measured from the $\operatorname{Ly} \alpha$ forest opacity at $z \approx 3$. Further study is needed to determine the nature of the LyC properties of these sources.

Recent measurements of the LBG LF at redshifts $z \gtrsim 6$ suggest a large value of $f_{\mathrm{esc}}^{\mathrm{LyC}}$ is needed with substantial contribution from relatively faint galaxies in order for the universe to be reionized by $z \sim 6$ (Ouchi et al. 2009; Oesch et al. 2009, 2010; Bouwens et al. 2010). While the extremely blue NB3640- $R$ colors of our faint LAE sample are difficult to explain within current theoretical models for the UV and LyC emission properties of star-forming regions, reconciling the observations with models is an important step toward understanding the processes involved in reionization. To this end, we are augmenting our data sets with additional $H S T$ imaging and ground-based spectra of both LBGs and LAEs with NB3640 detections. In addition to providing independent confirmation of LyC detections, such data are needed to confirm the redshifts of the LAEs. Finally we note that while our samples of LBGs and LAEs are large enough to determine robust average properties, it may be that the SSA22a protocluster is itself unique. Thus, we have ongoing projects to supplement this work with similar studies in other fields.

We thank Andrew Blain for permitting our use of the NB3640 filter, Kevin Hainline for his helpful discussions regarding our photometric simulations, and the anonymous referee for constructive suggestions. D.B.N. and A.E.S. acknowledge support from the David and Lucile Packard Foundation. C.C.S. acknowledges additional support from the John D. and Catherine T. MacArthur Foundation, the Peter and Patricia Gruber Foundation, and NSF grants AST-0606912 and AST-0908805. We wish to extend special thanks to those of Hawaiian ancestry on whose sacred mountain we are privileged to be guests. Without their generous hospitality, most of the observations presented herein would not have been possible.

\section{APPENDIX}

\section{LBGs AND LAEs WITH NO NB3640 DETECTION}

In this appendix we present coordinates, $R$-band magnitudes, and limits on the observed UV-to-LyC flux density ratios for the LBGs and LAEs that were undetected in the NB3640 image, as well as emission and interstellar absorption redshifts for the LBGs. Table 9 lists the LBGs and Table 10 lists the LAEs. The color $B V-\mathrm{NB} 4980=3.49$ corresponds to infinite Ly $\alpha$ equivalent widths; values near this limit reflect the large photometric uncertainties.

Table 9

Photometry for LBGs without NB3640 Detections

\begin{tabular}{lcccccc}
\hline \hline ID & R.A. & $\begin{array}{c}\text { Decl. } \\
(J 2000)\end{array}$ & $z_{\mathrm{em}}{ }^{\mathrm{a}}$ & $z_{\mathrm{abs}}{ }^{\mathrm{b}}$ & $R$ & $\frac{F_{\mathrm{UV}}{ }^{\mathrm{c}}}{{ }_{\mathrm{LyC}}}$ \\
& $(\mathrm{J} 200 \mathrm{~s})$ & & & & \\
\hline C32 & $22: 17: 25.63$ & $0: 16: 12.9$ & 3.301 & 3.290 & 23.64 & $>29.2$ \\
C30 & $22: 17: 19.29$ & $0: 15: 45.0$ & 3.104 & 3.097 & 23.70 & $>27.5$ \\
C47 & $22: 17: 20.24$ & $0: 17: 32.5$ & 3.075 & 3.065 & 23.78 & $>25.7$ \\
D3 & $22: 17: 32.40$ & $0: 11: 33.6$ & 3.086 & 3.077 & 23.92 & $>22.4$ \\
C35 & $22: 17: 20.23$ & $0: 16: 52.5$ & 3.103 & 3.098 & 24.06 & $>19.7$ \\
C24 & $22: 17: 18.94$ & $0: 14: 45.4$ & 3.102 & 3.091 & 24.19 & $>17.6$ \\
C11 & $22: 17: 25.68$ & $0: 12: 35.4$ & 3.109 & 3.099 & 24.21 & $>17.3$ \\
C12 & $22: 17: 35.29$ & $0: 12: 47.9$ & 3.118 & 3.106 & 24.22 & $>17.0$ \\
C4 & $22: 17: 38.91$ & $0: 11: 02.0$ & 3.076 & $\ldots$ & 24.28 & $>16.2$ \\
MD23 & $22: 17: 28.01$ & $0: 14: 29.6$ & 3.092 & 3.082 & 24.34 & $>15.2$ \\
MD14 & $22: 17: 37.91$ & $0: 13: 43.9$ & $\ldots$ & 3.094 & 24.49 & $>13.4$ \\
M10 & $22: 17: 26.80$ & $0: 12: 21.3$ & 3.103 & 3.095 & 24.50 & $>13.1$ \\
C48 & $22: 17: 18.58$ & $0: 18: 16.7$ & 3.090 & 3.079 & 24.57 & $>12.3$ \\
M28 & $22: 17: 31.66$ & $0: 16: 58.0$ & 3.094 & 3.088 & 24.75 & $>10.4$ \\
C28 & $22: 17: 21.13$ & $0: 15: 27.7$ & 3.076 & $\ldots$ & 24.87 & $>9.3$ \\
C50 & $22: 17: 37.68$ & $0: 18: 21.2$ & $\ldots$ & 3.086 & 25.01 & $>8.2$ \\
C26 & $22: 17: 39.54$ & $0: 15: 15.6$ & 3.178 & $\ldots$ & 25.01 & $>8.2$ \\
C15 & $22: 17: 26.13$ & $0: 12: 55.4$ & 3.094 & $\ldots$ & 25.02 & $>8.2$ \\
C39 & $22: 17: 20.99$ & $0: 17: 09.5$ & 3.076 & $\ldots$ & 25.04 & $>8.0$ \\
M14 & $22: 17: 39.05$ & $0: 13: 30.1$ & 3.091 & $\ldots$ & 25.20 & $>6.9$ \\
\hline
\end{tabular}

Notes.

${ }^{a}$ Ly $\alpha$ emission redshift.

b Interstellar absorption redshift.

${ }^{c}$ Lower limit for observed ratio of non-ionizing UV and LyC emission with no correction for IGM absorption. 
Table 10

Photometry for LAEs without NB3640 Detections

\begin{tabular}{|c|c|c|c|c|c|c|c|}
\hline ID & $\begin{array}{c}\text { R.A. } \\
\text { (J2000) }\end{array}$ & $\begin{array}{c}\text { Decl. } \\
(\mathrm{J} 2000)\end{array}$ & 4980 & $B V-\mathrm{NB} 4980$ & $\begin{array}{c}\mathrm{EW}_{0}{ }^{\mathrm{a}} \\
(\AA)\end{array}$ & $R$ & $\frac{F_{\mathrm{UV}}}{F_{\mathrm{LyC}}}{ }_{\mathrm{obs}}{ }^{\mathrm{b}}$ \\
\hline $001^{\mathrm{c}}$ & $22: 17: 32.40$ & $0: 11: 34.1$ & 23.00 & 0.74 & 21 & 23.92 & $>22.4$ \\
\hline $002^{\mathrm{d}}$ & $22: 17: 38.90$ & $0: 11: 01.8$ & 23.15 & 1.41 & 61 & 24.28 & $>16.2$ \\
\hline $004^{\mathrm{e}}$ & $22: 17: 28.01$ & $0: 14: 30.0$ & 23.40 & 1.06 & 36 & 24.34 & $>15.2$ \\
\hline 005 & $22: 17: 35.86$ & $0: 15: 59.4$ & 23.74 & 2.68 & 400 & 25.65 & $>4.6$ \\
\hline 006 & $22: 17: 24.80$ & $0: 11: 16.8$ & 23.76 & 1.69 & 90 & $>27$ & $\ldots$ \\
\hline 007 & $22: 17: 27.78$ & $0: 17: 36.9$ & 23.84 & 1.96 & 131 & $>27$ & $\ldots$ \\
\hline $008^{\mathrm{f}}$ & $22: 17: 21.11$ & $0: 15: 28.0$ & 24.00 & 0.95 & 30 & 24.87 & $>9.3$ \\
\hline $009^{\mathrm{g}}$ & $22: 17: 28.29$ & $0: 12: 12.3$ & 24.02 & 1.66 & 87 & 25.84 & $>3.8$ \\
\hline 011 & $22: 17: 33.85$ & $0: 12: 14.9$ & 24.15 & 1.54 & 73 & 26.07 & $>3.1$ \\
\hline $012^{\mathrm{h}}$ & $22: 17: 31.69$ & $0: 16: 57.6$ & 24.23 & 0.97 & 31 & 24.75 & $>10.5$ \\
\hline 013 & $22: 17: 27.18$ & $0: 16: 21.7$ & 24.23 & 1.91 & 122 & 25.98 & $>3.4$ \\
\hline 014 & $22: 17: 19.25$ & $0: 14: 50.9$ & 24.27 & 1.42 & 62 & 25.82 & $>3.9$ \\
\hline 015 & $22: 17: 21.84$ & $0: 12: 12.7$ & 24.28 & 1.07 & 37 & $>27$ & $\ldots$ \\
\hline 017 & $22: 17: 25.40$ & $0: 17: 16.8$ & 24.43 & 2.14 & 170 & 26.22 & $>2.7$ \\
\hline 020 & $22: 17: 37.33$ & $0: 16: 31.4$ & 24.55 & 1.44 & 64 & 25.45 & $>5.5$ \\
\hline 022 & $22: 17: 19.68$ & $0: 11: 49.4$ & 24.58 & 1.80 & 105 & 26.11 & $>3.0$ \\
\hline 023 & $22: 17: 31.73$ & $0: 16: 06.9$ & 24.61 & 0.88 & 27 & 24.91 & $>9.1$ \\
\hline 024 & $22: 17: 34.17$ & 0:16:09.7 & 24.68 & 2.11 & 162 & 26.73 & $>1.7$ \\
\hline 026 & $22: 17: 18.96$ & $0: 12: 00.8$ & 24.70 & 1.63 & 83 & 26.59 & $>1.9$ \\
\hline 027 & $22: 17: 24.94$ & $0: 17: 17.3$ & 24.74 & 1.59 & 79 & 26.25 & $>2.6$ \\
\hline $029^{\mathrm{i}}$ & $22: 17: 31.49$ & $0: 12: 55.0$ & 24.77 & 0.82 & 24 & 25.38 & $>5.9$ \\
\hline 030 & $22: 17: 21.75$ & $0: 11: 38.8$ & 24.81 & 1.34 & 55 & $>27$ & $\ldots$ \\
\hline 031 & $22: 17: 33.63$ & $0: 17: 15.1$ & 24.83 & 1.67 & 88 & 26.37 & $>2.3$ \\
\hline 032 & $22: 17: 26.61$ & $0: 13: 18.1$ & 24.83 & 1.17 & 43 & 26.61 & $>1.9$ \\
\hline 033 & $22: 17: 37.50$ & 0:14:08.3 & 24.85 & 1.58 & 78 & 26.62 & $>1.9$ \\
\hline 035 & $22: 17: 27.03$ & $0: 13: 13.2$ & 24.87 & 0.90 & 28 & $>27$ & $\ldots$ \\
\hline 036 & $22: 17: 22.25$ & $0: 11: 55.1$ & 24.89 & 1.85 & 113 & $>27$ & $\ldots$ \\
\hline 037 & $22: 17: 20.96$ & 0:18:07.3 & 24.89 & 0.97 & 31 & 25.61 & $>4.7$ \\
\hline 040 & $22: 17: 31.93$ & 0:13:08.5 & 24.92 & 1.96 & 131 & $>27$ & $\ldots$ \\
\hline 042 & $22: 17: 21.50$ & $0: 17: 04.7$ & 24.93 & 1.04 & 35 & 25.50 & $>5.2$ \\
\hline 043 & $22: 17: 21.65$ & $0: 12: 23.4$ & 24.98 & 1.30 & 52 & 26.24 & $>2.7$ \\
\hline 044 & $22: 17: 36.41$ & $0: 12: 51.0$ & 24.99 & 1.36 & 57 & $>27$ & $\ldots$ \\
\hline 045 & $22: 17: 35.97$ & $0: 16: 30.2$ & 25.03 & 1.45 & 65 & $>27$ & $\ldots$ \\
\hline 047 & $22: 17: 36.05$ & $0: 15: 06.9$ & 25.04 & 1.96 & 131 & $>27$ & $\ldots$ \\
\hline 049 & $22: 17: 39.29$ & $0: 16: 10.5$ & 25.06 & 2.17 & 177 & $>27$ & $\ldots$ \\
\hline 050 & $22: 17: 24.56$ & $0: 15: 56.8$ & 25.08 & $>2.42$ & $>258$ & $>27$ & $\ldots$ \\
\hline 052 & $22: 17: 36.84$ & $0: 13: 17.2$ & 25.13 & 1.04 & 35 & 26.63 & $>1.8$ \\
\hline 054 & $22: 17: 39.05$ & $0: 11: 33.9$ & 25.18 & 1.85 & 113 & 26.25 & $>2.6$ \\
\hline 055 & $22: 17: 35.80$ & $0: 11: 50.0$ & 25.20 & 1.81 & 107 & 26.67 & $>1.8$ \\
\hline 056 & $22: 17: 22.42$ & $0: 17: 20.7$ & 25.21 & 1.75 & 98 & 26.86 & $>1.5$ \\
\hline 057 & $22: 17: 25.40$ & $0: 10: 58.3$ & 25.23 & 1.07 & 37 & 26.84 & $>1.5$ \\
\hline 058 & $22: 17: 19.61$ & $0: 15: 38.4$ & 25.27 & 1.44 & 64 & $>27$ & $\ldots$ \\
\hline 059 & $22: 17: 24.98$ & $0: 12: 30.0$ & 25.29 & 0.85 & 25 & 25.31 & $>6.2$ \\
\hline 060 & $22: 17: 28.19$ & $0: 11: 17.1$ & 25.30 & 1.16 & 42 & 26.61 & $>1.9$ \\
\hline 061 & $22: 17: 34.10$ & $0: 15: 40.2$ & 25.31 & $>2.19$ & $>182$ & $>27$ & $\ldots$ \\
\hline 062 & $22: 17: 22.87$ & $0: 14: 41.7$ & 25.31 & 1.26 & 49 & 26.53 & $>2.0$ \\
\hline 063 & $22: 17: 23.32$ & $0: 15: 52.9$ & 25.31 & 1.63 & 83 & 26.55 & $>2.0$ \\
\hline 065 & $22: 17: 28.15$ & $0: 14: 36.4$ & 25.38 & $>2.12$ & $>165$ & 26.91 & $>1.4$ \\
\hline 066 & $22: 17: 20.86$ & $0: 15: 11.8$ & 25.41 & 0.73 & 20 & 26.64 & $>1.8$ \\
\hline 067 & $22: 17: 36.26$ & $0: 13: 11.7$ & 25.42 & 1.13 & 40 & 26.40 & $>2.3$ \\
\hline 068 & $22: 17: 18.37$ & $0: 17: 26.1$ & 25.44 & 1.28 & 51 & $>27$ & $\ldots$ \\
\hline 070 & $22: 17: 39.28$ & 0:14:00.2 & 25.45 & 0.78 & 22 & 25.98 & $>3.4$ \\
\hline 071 & $22: 17: 21.61$ & $0: 12: 20.5$ & 25.48 & 1.23 & 47 & 26.77 & $>1.6$ \\
\hline 072 & $22: 17: 31.24$ & $0: 17: 32.1$ & 25.48 & 1.78 & 102 & 27.00 & $>1.3$ \\
\hline 073 & $22: 17: 39.12$ & $0: 17: 11.7$ & 25.50 & 1.98 & 135 & 26.35 & $>2.4$ \\
\hline 075 & $22: 17: 22.97$ & $0: 11: 25.8$ & 25.51 & $>1.99$ & $>137$ & $>27$ & $\ldots$ \\
\hline 076 & $22: 17: 20.67$ & $0: 15: 13.2$ & 25.54 & 1.13 & 40 & $>27$ & $\ldots$ \\
\hline 078 & $22: 17: 37.68$ & $0: 16: 48.3$ & 25.56 & 1.21 & 46 & 25.95 & $>3.5$ \\
\hline 079 & $22: 17: 34.68$ & $0: 11: 10.5$ & 25.56 & 2.19 & 182 & $>27$ & $\ldots$ \\
\hline 080 & $22: 17: 35.95$ & $0: 13: 43.3$ & 25.58 & 1.33 & 54 & $>27$ & $\ldots$ \\
\hline 082 & $22: 17: 35.44$ & $0: 16: 47.6$ & 25.60 & 1.53 & 72 & $>27$ & $\ldots$ \\
\hline 085 & $22: 17: 30.86$ & $0: 14: 38.2$ & 25.65 & 1.16 & 42 & 26.94 & $>1.4$ \\
\hline 086 & $22: 17: 28.42$ & $0: 13: 42.8$ & 25.65 & $>1.85$ & $>113$ & $>27$ & $\ldots$ \\
\hline 088 & $22: 17: 38.45$ & $0: 13: 18.3$ & 25.74 & 1.25 & 48 & $>27$ & $\ldots$ \\
\hline 089 & $22: 17: 38.54$ & $0: 15: 22.5$ & 25.74 & $>1.76$ & $>100$ & 26.59 & $>1.9$ \\
\hline
\end{tabular}




\begin{tabular}{|c|c|c|c|c|c|c|c|}
\hline \multicolumn{8}{|c|}{$\begin{array}{c}\text { Table 10 } \\
\text { (Continued) }\end{array}$} \\
\hline ID & $\begin{array}{c}\text { R.A. } \\
\text { (J2000) }\end{array}$ & $\begin{array}{c}\text { Decl. } \\
(\mathrm{J} 2000)\end{array}$ & 4980 & $B V-\mathrm{NB} 4980$ & $\begin{array}{c}\mathrm{EW}_{0}{ }^{\mathrm{a}} \\
(\AA)\end{array}$ & $R$ & $\begin{array}{c}F_{\mathrm{UV}} \\
F_{\mathrm{LyC}} \\
\text { obs }\end{array}$ \\
\hline 090 & $22: 17: 18.25$ & $0: 14: 06.4$ & 25.75 & 1.30 & 52 & $>27$ & $\ldots$ \\
\hline 091 & $22: 17: 36.14$ & $0: 15: 40.7$ & 25.78 & $>1.72$ & $>94$ & $>27$ & $\ldots$ \\
\hline 092 & $22: 17: 23.97$ & $0: 15: 27.8$ & 25.79 & $>1.71$ & $>93$ & $>27$ & $\ldots$ \\
\hline 093 & $22: 17: 27.48$ & $0: 13: 57.5$ & 25.80 & 0.78 & 22 & 26.35 & $>2.4$ \\
\hline 094 & $22: 17: 39.14$ & $0: 17: 00.6$ & 25.82 & $>1.68$ & $>89$ & $>27$ & $\ldots$ \\
\hline 095 & $22: 17: 37.19$ & $0: 13: 28.0$ & 25.82 & 1.13 & 40 & 26.17 & $>2.8$ \\
\hline 097 & $22: 17: 27.11$ & $0: 14: 08.7$ & 25.85 & 0.98 & 32 & $>27$ & $\ldots$ \\
\hline 098 & $22: 17: 24.01$ & $0: 13: 19.5$ & 25.86 & $>1.64$ & $>84$ & $>27$ & $\ldots$ \\
\hline 099 & $22: 17: 36.46$ & $0: 13: 00.3$ & 25.87 & $>1.63$ & $>83$ & $>27$ & $\ldots$ \\
\hline 100 & $22: 17: 30.61$ & $0: 18: 11.6$ & 25.89 & 1.26 & 49 & $>27$ & $\ldots$ \\
\hline 103 & $22: 17: 19.40$ & $0: 15: 26.1$ & 25.93 & $>1.57$ & $>76$ & $>27$ & $\ldots$ \\
\hline 104 & $22: 17: 37.66$ & $0: 12: 55.5$ & 25.94 & $>1.56$ & $>75$ & $>27$ & $\ldots$ \\
\hline 105 & $22: 17: 35.46$ & $0: 12: 23.9$ & 25.94 & 0.81 & 24 & 26.81 & $>1.6$ \\
\hline 106 & $22: 17: 22.86$ & $0: 17: 57.8$ & 25.96 & 1.54 & 73 & 26.16 & $>2.9$ \\
\hline 107 & $22: 17: 20.96$ & $0: 14: 46.7$ & 25.97 & 0.76 & 22 & 26.57 & $>2.0$ \\
\hline 108 & $22: 17: 24.78$ & $0: 17: 40.4$ & 25.97 & 1.02 & 34 & 26.84 & $>1.5$ \\
\hline 109 & $22: 17: 23.98$ & $0: 17: 57.8$ & 25.99 & 0.77 & 22 & 26.77 & $>1.6$ \\
\hline 110 & $22: 17: 19.50$ & $0: 15: 57.6$ & 25.99 & $>1.51$ & $>70$ & $>27$ & $\ldots$ \\
\hline 111 & $22: 17: 31.14$ & $0: 16: 42.9$ & 26.07 & $>1.44$ & $>64$ & $>27$ & $\ldots$ \\
\hline 112 & $22: 17: 32.72$ & $0: 15: 54.2$ & 26.07 & $>1.44$ & $>64$ & $>27$ & $\cdots$ \\
\hline 113 & $22: 17: 24.80$ & $0: 13: 26.9$ & 26.07 & $>1.44$ & $>64$ & $>27$ & $\ldots$ \\
\hline 114 & $22: 17: 34.50$ & $0: 14: 20.0$ & 26.08 & $>1.44$ & $>64$ & $>27$ & $\ldots$ \\
\hline 115 & $22: 17: 33.46$ & $0: 17: 01.2$ & 26.09 & $>1.43$ & $>63$ & $>27$ & $\ldots$ \\
\hline 116 & $22: 17: 28.00$ & $0: 12: 14.2$ & 26.10 & $>1.43$ & $>63$ & $>27$ & $\ldots$ \\
\hline 117 & $22: 17: 39.08$ & $0: 12: 01.9$ & 26.11 & $>1.42$ & $>62$ & $>27$ & $\ldots$ \\
\hline 119 & $22: 17: 25.63$ & $0: 12: 47.8$ & 26.18 & $>1.38$ & $>59$ & $>27$ & $\ldots$ \\
\hline 120 & $22: 17: 26.76$ & $0: 10: 59.8$ & 26.25 & $>1.35$ & $>56$ & $>27$ & $\ldots$ \\
\hline 121 & $22: 17: 26.44$ & $0: 15: 27.5$ & 26.30 & $>1.33$ & $>54$ & $>27$ & $\ldots$ \\
\hline 122 & $22: 17: 38.19$ & $0: 14: 03.7$ & 26.30 & $>1.32$ & $>54$ & $>27$ & $\ldots$ \\
\hline 123 & $22: 17: 35.06$ & $0: 17: 26.0$ & 26.31 & $>1.32$ & $>54$ & $>27$ & $\ldots$ \\
\hline 124 & $22: 17: 22.80$ & $0: 17: 48.7$ & 26.32 & $>1.32$ & $>54$ & $>27$ & $\cdots$ \\
\hline 125 & $22: 17: 38.02$ & $0: 14: 03.6$ & 26.32 & $>1.31$ & $>53$ & 26.79 & $>1.6$ \\
\hline 126 & $22: 17: 19.53$ & $0: 16: 48.2$ & 26.33 & $>1.31$ & $>53$ & $>27$ & $\ldots$ \\
\hline 127 & $22: 17: 36.91$ & $0: 11: 27.1$ & 26.36 & $>1.29$ & $>51$ & $>27$ & $\ldots$ \\
\hline 128 & $22: 17: 23.43$ & $0: 16: 07.4$ & 26.48 & $>1.23$ & $>47$ & $>27$ & $\ldots$ \\
\hline 129 & $22: 17: 22.28$ & $0: 10: 57.9$ & 26.49 & $>1.23$ & $>47$ & $>27$ & $\ldots$ \\
\hline 130 & $22: 17: 32.84$ & $0: 16: 48.8$ & 26.49 & $>1.23$ & $>47$ & $>27$ & $\ldots$ \\
\hline
\end{tabular}

\section{Notes.}

${ }^{a}$ Ly $\alpha$ rest equivalent width estimated from $B V-\mathrm{NB} 4980$ color.

${ }^{b}$ Lower limit for observed ratio of non-ionizing UV and LyC emission with no correction for IGM absorption.

${ }^{\mathrm{c}} \mathrm{LBG} \mathrm{D} 3, z_{\mathrm{em}}=3.086$.

${ }^{\mathrm{d}} \mathrm{LBG} \mathrm{C} 4, z_{\mathrm{em}}=3.076$.

${ }^{\mathrm{e}} \mathrm{LBG}$ MD23, $z_{\mathrm{em}}=3.092$.

${ }^{\mathrm{f}} \mathrm{LBG} \mathrm{C28,} z_{\mathrm{em}}=3.076$.

g LBG candidate C9.

${ }^{\mathrm{h}}$ LBG M28, $z_{\mathrm{em}}=3.094$.

${ }^{\mathrm{i}}$ LBG candidate M13.

\section{REFERENCES}

Abraham, R. G., et al. 2007, ApJ, 669, 184

Ando, M., Ohta, K., Iwata, I., Akiyama, M., Aoki, K., \& Tamura, N. 2006, ApJ, 645, L9

Arnouts, S., et al. 2005, ApJ, 619, L43

Baba, H., et al. 2002, in ASP Conf. Ser. 281, Astronomical Data Analysis Software and Systems XI, ed. D. A. Bohlender, D. Durand, \& T. H. Handley (San Francisco, CA: ASP), 298

Becker, G. D., Rauch, M., \& Sargent, W. L. W. 2007, ApJ, 662, 72

Becker, G. D., Sargent, W. L. W., \& Rauch, M. 2004, ApJ, 613, 61

Bertin, E., \& Arnouts, S. 1996, A\&AS, 117, 393

Bolton, J. S., \& Haehnelt, M. G. 2007, MNRAS, 382, 325

Bolton, J. S., Haehnelt, M. G., Viel, M., \& Springel, V. 2005, MNRAS, 357, 1178
Bouwens, R. J., et al. 2010, arXiv:1006.4360

Bridge, C. R., et al. 2010, ApJ, 720, 465

Bunker, A. J., et al. 2010, MNRAS, 409, 855

Casey, C. M., Impey, C. D., Petry, C. E., Marble, A. R., \& Davé, R. 2008, AJ, 136,181

Chen, H., Prochaska, J. X., \& Gnedin, N. Y. 2007, ApJ, 667, L125

Cowie, L. L., Barger, A. J., \& Trouille, L. 2009, ApJ, 692, 1476

Fan, X., et al. 2006, AJ, 132, 117

Faucher-Giguère, C., Lidz, A., Hernquist, L., \& Zaldarriaga, M. 2008, ApJ, 688, 85

Gawiser, E., et al. 2007, ApJ, 671, 278

Geach, J. E., et al. 2009, ApJ, 700, 1

Gnedin, N. Y. 2008, ApJ, 673, L1

Gnedin, N. Y., Kravtsov, A. V., \& Chen, H. 2008, ApJ, 672, 765

Grimes, J. P., et al. 2009, ApJS, 181, 272 
Hayashino, T., et al. 2004, AJ, 128, 2073

Hopkins, P. F., Richards, G. T., \& Hernquist, L. 2007, ApJ, 654, 731

Hu, E. M., Cowie, L. L., Capak, P., McMahon, R. G., Hayashino, T., \& Komiyama, Y. 2004, AJ, 127, 563

Inoue, A. K. 2010, MNRAS, 401, 1325

Inoue, A. K., \& Iwata, I. 2008, MNRAS, 387, 1681

Inoue, A. K., et al. 2011, MNRAS, 411, 2336

Iwata, I., et al. 2009, ApJ, 692, 1287

Kirkman, D., et al. 2005, MNRAS, 360, 1373

Landolt, A. U. 1992, AJ, 104, 340

Law, D. R., Steidel, C. C., Erb, D. K., Pettini, M., Reddy, N. A., Shapley, A. E., Adelberger, K. L., \& Simenc, D. J. 2007, ApJ, 656, 1

Lotz, J. M., Madau, P., Giavalisco, M., Primack, J., \& Ferguson, H. C. 2006, ApJ, 636, 592

Malkan, M., Webb, W., \& Konopacky, Q. 2003, ApJ, 598, 878

Massey, P., Strobel, K., Barnes, J. V., \& Anderson, E. 1988, ApJ, 328, 315

Matsuda, Y., et al. 2004, AJ, 128, 569

Meiksin, A. A. 2009, Rev. Mod. Phys., 81, 1405

Miyazaki, S., et al. 2002, PASJ, 54, 833

Nonino, M., et al. 2009, ApJS, 183, 244

Oesch, P. A., et al. 2009, ApJ, 690, 1350

Oesch, P. A., et al. 2010, ApJ, 709, L16

Ono, Y., et al. 2010, MNRAS, 402, 1580

Ouchi, M., et al. 2004, ApJ, 611, 660

Ouchi, M., et al. 2009, ApJ, 706, 1136

Peter, A. H. G., Shapley, A. E., Law, D. R., Steidel, C. C., Erb, D. K., Reddy, N. A., \& Pettini, M. 2007, ApJ, 668, 23

Prochaska, J. X., O’Meara, J. M., \& Worseck, G. 2010, ApJ, 718, 392

Prochaska, J. X., Worseck, G., \& O’Meara, J. M. 2009, ApJ, 705, L113

Razoumov, A. O., \& Sommer-Larsen, J. 2007, ApJ, 668, 674

Reddy, N. A., \& Steidel, C. C. 2009, ApJ, 692, 778
Reddy, N. A., Steidel, C. C., Pettini, M., Adelberger, K. L., Shapley, A. E., Erb, D. K., \& Dickinson, M. 2008, ApJS, 175, 48

Robertson, B. E., Ellis, R. S., Dunlop, J. S., McLure, R. J., \& Stark, D. P. 2010, Nature, 468, 49

Schlegel, D. J., Finkbeiner, D. P., \& Davis, M. 1998, ApJ, 500, 525

Shapley, A. E., Steidel, C. C., Pettini, M., \& Adelberger, K. L. 2003, ApJ, 588 , 65

Shapley, A. E., Steidel, C. C., Pettini, M., Adelberger, K. L., \& Erb, D. K. 2006, ApJ, 651, 688

Siana, B., et al. 2007, ApJ, 668, 62

Siana, B., et al. 2010, ApJ, 723, 241

Songaila, A., \& Cowie, L. L. 2010, ApJ, 721, 1448

Stark, D. P., Ellis, R. S., Chiu, K., Ouchi, M., \& Bunker, A. 2010, MNRAS, 408,1628

Steidel, C. C. 1992 , PASP, 104, 843

Steidel, C. C., Adelberger, K. L., Dickinson, M., Giavalisco, M., Pettini, M., \& Kellogg, M. 1998, ApJ, 492, 428

Steidel, C. C., Adelberger, K. L., Shapley, A. E., Pettini, M., Dickinson, M., \& Giavalisco, M. 2000, ApJ, 532, 170

Steidel, C. C., Adelberger, K. L., Shapley, A. E., Pettini, M., Dickinson, M., \& Giavalisco, M. 2003, ApJ, 592, 728

Steidel, C. C., Bogosavljević, M., Shapley, A. E., Kollmeier, J. A., Reddy, N. A., Erb, D. K., \& Pettini, M. 2011, arXiv:1101.2204

Steidel, C. C., Pettini, M., \& Adelberger, K. L. 2001, ApJ, 546, 665

Steidel, C. C., Shapley, A. E., Pettini, M., Adelberger, K. L., Erb, D. K., Reddy, N. A., \& Hunt, M. P. 2004, ApJ, 604, 534

Stengler-Larrea, E. A., et al. 1995, ApJ, 444, 64

Vanzella, E., Siana, B., Cristiani, S., \& Nonino, M. 2010a, MNRAS, 404, 1672

Vanzella, E., et al. 2010b, ApJ, 725, 1011

Yagi, M., Kashikawa, N., Sekiguchi, M., Doi, M., Yasuda, N., Shimasaku, K., \& Okamura, S. 2002, AJ, 123, 66 